TITLE:

Hydrogen transport diagnostics by atomic and molecular emission line profiles simultaneously measured for large helical device

$\operatorname{AUTHOR}(S)$ :

Fujii, K.; Shikama, T.; Goto, M.; Morita, S.; Hasuo, M.

CITATION:

Fujii, K.... [et al]. Hydrogen transport diagnostics by atomic and molecular emission line profiles simultaneously measured for large helical device. Physics of Plasmas 2013, 20(1): 012514.

ISSUE DATE:

2013-01

URL:

http://hdl.handle.net/2433/171236

RIGHT:

(C) 2013 American Institute of Physics 


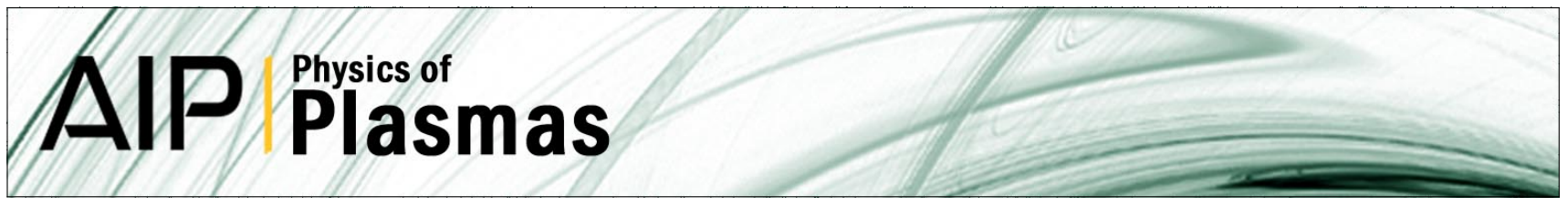

\section{Hydrogen transport diagnostics by atomic and molecular emission line profiles simultaneously measured for large helical device}

K. Fujii, T. Shikama, M. Goto, S. Morita, and M. Hasuo

Citation: Phys. Plasmas 20, 012514 (2013); doi: 10.1063/1.4789454

View online: http://dx.doi.org/10.1063/1.4789454

View Table of Contents: http://pop.aip.org/resource/1/PHPAEN/v20/i1

Published by the American Institute of Physics.

\section{Related Articles}

Experimental investigation of the ribbon-array ablation process

Phys. Plasmas 20, 032702 (2013)

Rotating copper plasmoid in external magnetic field

Phys. Plasmas 20, 022117 (2013)

Thomson parabola spectrometry for gold laser-generated plasmas

Phys. Plasmas 20, 023106 (2013)

Evidence for hydrogen generation in laser- or spark-induced cavitation bubbles

Appl. Phys. Lett. 102, 074105 (2013)

Oblique shock structures formed during the ablation phase of aluminium wire array z-pinches

Phys. Plasmas 20, 022705 (2013)

\section{Additional information on Phys. Plasmas}

Journal Homepage: http://pop.aip.org/

Journal Information: http://pop.aip.org/about/about_the_journal

Top downloads: http://pop.aip.org/features/most_downloaded

Information for Authors: http://pop.aip.org/authors

\section{ADVERTISEMENT}

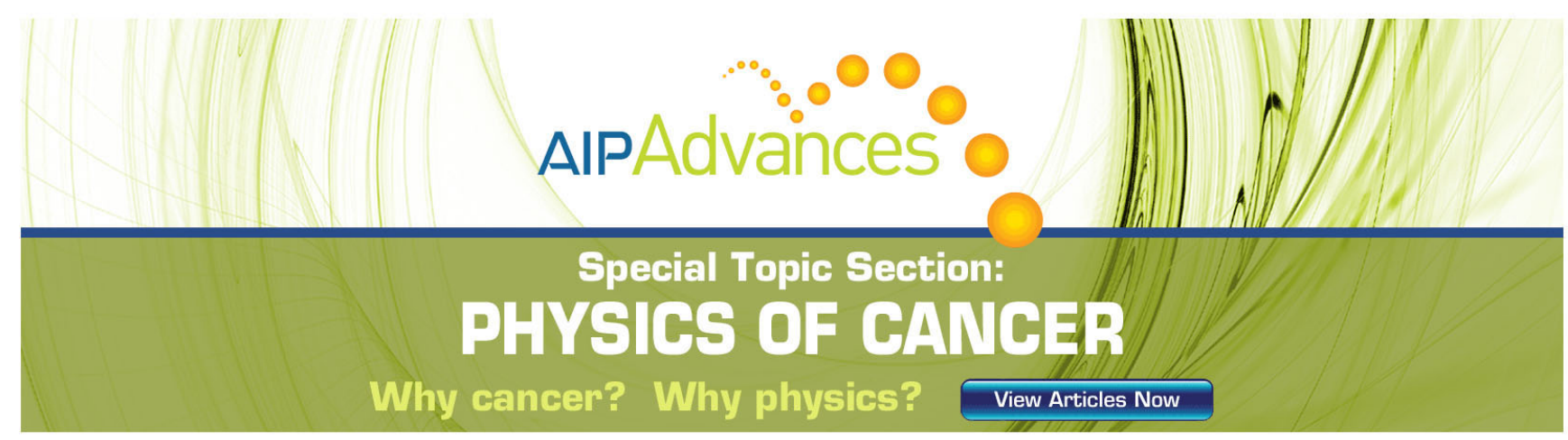




\title{
Hydrogen transport diagnostics by atomic and molecular emission line profiles simultaneously measured for large helical device
}

\author{
K. Fujii, ${ }^{1, a)}$ T. Shikama, ${ }^{1}$ M. Goto, ${ }^{2}$ S. Morita, ${ }^{2}$ and M. Hasuo ${ }^{1}$ \\ ${ }^{1}$ Department of Mechanical Engineering and Science, Graduate School of Engineering, Kyoto University, \\ Kyoto 606-8501, Japan \\ ${ }^{2}$ National Institute for Fusion Science, Toki 509-5292, Japan
}

(Received 4 October 2012; accepted 8 January 2013; published online 29 January 2013)

\begin{abstract}
We observe the Balmer- $\alpha,-\beta$, and $-\gamma$ lines of hydrogen atoms and $Q$ branches of the Fulcher- $\alpha$ band of hydrogen molecules simultaneously with their polarization resolved for large helical device. From the fit including the line splits and the polarization dependences by the Zeeman effect, the emission locations, intensities, and the temperatures of the atoms and molecules are determined. The emission locations of the hydrogen atoms are determined outside but close to the last closed flux surface (LCFS). The results are consistent with a previous work (Phys. Plasmas 12, 042501 (2005)). On the other hand, the emission locations of the molecules are determined to be in the divertor legs, which is farer from those of the atoms. The kinetic energy of the atoms is $1 \sim 20 \mathrm{eV}$, while the rotational temperature of molecules is $\sim 0.04 \mathrm{eV}$. Additionally, substantial wings, which originate from high velocity atoms and are not reproduced by the conventional spectral analysis, are observed in the Balmer line profiles. We develop a one-dimensional model to simulate the transport of the atoms and molecules. The model reproduces the differences of the emission locations of the atoms and molecules when their initial temperatures are assumed to be $3 \mathrm{eV}$ and $0.04 \mathrm{eV}$, respectively. From the model, the wings of the Balmer- $\alpha$ line is attributed to the high velocity atoms exist deep inside the LCFS, which are generated by the charge exchange collisions with hot protons there. (C) 2013 American Institute of Physics. [http://dx.doi.org/10.1063/1.4789454]
\end{abstract}

\section{INTRODUCTION}

In peripheral regions of magnetically plasma confinement devices, neutral hydrogen molecules and atoms are generated from desorption or recombination on the first walls. Some of them travel toward the plasma and are ionized by electron impacts. Since the outflux of the charged particles from the confined region and the ionization flux of the hydrogen atoms and molecules balance in a stable plasma, the dynamics of the neutrals have a great influence on the confinement performance. ${ }^{1,2}$ For establishing stable and a high-performance plasma, it is important to understand and control their dynamics.

Since atoms and molecules are excited and ionized in the plasma, the dynamics have been frequently observed by emission measurements. ${ }^{3,4}$ In simple emission measurements, the line integrated intensity is observed and no spatial information along the line of sight (LOS) is usually obtained. However, Weaver et al. have proposed a method to separate the emissions into two points along the line of sight and determine the emission locations of the Balmer- $\alpha$ line from comparisons of its Zeeman split and the spatial distributions of the magnetic field along the LOS. ${ }^{5}$ The method has been expanded to multiple LOSs measurements for more spatial information by Shikama et al. in TRIAM-1 M tokamak. ${ }^{6}$

Iwamae et al. combines a polarization-resolved spectroscopic technique with the Zeeman split analysis method for increasing the measurement accuracies. ${ }^{7,8}$ They have meas-

\footnotetext{
a)fujii@me.kyoto-u.ac.jp.
}

ured the Balmer- $\alpha$ line shape for large helical device (LHD) with eight LOSs with their polarization resolved. They determined the emission locations of the Balmer- $\alpha$ line from the polarization dependence of the line shapes by the Zeeman split. The reported emission locations of the Balmer- $\alpha$ lines are almost outside the last closed flux surface (LCFS) of LHD. $^{8}$

A relation between the Balmer- $\alpha$ line intensity and the ionization flux of the atoms is calculated by a collisionalradiative model, which has been developed by Sawada et al..$^{9-11}$ The emission intensity ratios of the Balmer- $\alpha,-\beta$, and $-\gamma$ lines have been predicted from the calculation to have a dependence on the electron temperature and density at the emission locations. A plasma diagnostic method using the emission intensity ratios have also been proposed. ${ }^{9}$

On the other hand, emission lines of hydrogen molecules in fusion devices have been observed by several groups. ${ }^{12,13}$ The relation between the emission intensities in the Fulcher$\alpha$ band and ionization/dissociation flux has been reported by Fantz et al. ${ }^{13}$ Vibrational and rotational temperatures of the molecules have also been determined by the several emission lines in the Fulcher- $\alpha$ band. ${ }^{14,15}$ However, the spatial resolved measurement using Zeeman splits have not been applied on the molecular emission lines.

Recently, we have developed a multi-wavelength-range high-resolution $(\mathrm{MH})$ spectrometer and enabled a simultaneous high-resolution observation of the Balmer- $\alpha,-\beta,-\gamma$ lines, and also the emission lines of the $Q$ branches in the Fulcher$\alpha$ band. $^{16,17}$ Here, we report on the measurement results of these emission lines for LHD. 


\section{EXPERIMENTAL SETUP}

Figure 1 shows a top view of LHD. In the figure, schematic illustrations of the plasma, neutral beam injectors (NBIs) for the plasma heating, a laser path for the Thomson scattering measurement, ${ }^{18}$ and an LOS for an emission measurement are shown. A hydrogen plasma with a helical shape is generated. The major radius of the plasma axis, $R_{\mathrm{ax}}$, and magnetic field strength at the axis, $B_{\mathrm{ax}}$, are $3.6 \mathrm{~m}$ and $2.71 \mathrm{~T}$, respectively.

Poloidal cross sections of the plasma along the laser path and the LOS are shown in Figures 2(a) and 3(a), respectively. Because of the helical shape of LHD, the profiles of these two cross sections in the toroidally distant locations are slightly different. The gray ellipsoidal curves show the magnetic flux surfaces of LHD, which show a contour of the pressure of the confined plasma. The outmost surface is the LCFS. Normalized radius, $\rho$, is defined as a measure of the magnetic flux surfaces, where 0 is assigned for the plasma axis and 1 for the LCFS. The $\rho$ along the laser path is shown in the middle axis in Figure 2(b). The gray areas are aggregates of the open magnetic field lines whose connection lengths are longer than $24.5 \mathrm{~m}$. The gray area which encircles the LCFS is called as "ergodic layer" and which connects the layer and the divertors are called as "divertor legs." Charged particles escaping from the confined region travel along the open magnetic field lines toward the divertor plates through the divertor legs.

Figure 2(b) shows the profiles of electron temperature, $T_{\mathrm{e}}$, and density, $n_{\mathrm{e}}$, measured by the Thomson scattering method $^{18}$ for a hydrogen plasma. The temperatures on the plasma axis and on the LCFS are several $\mathrm{keV}$ and several hundreds of eV, respectively. The density is in the order of $10^{19} \mathrm{~m}^{-3}$ inside the LCFS. The $T_{\mathrm{e}}$ and $n_{\mathrm{e}}$ drop sharply in the ergodic layer.

The plasma parameters inside the LCFS are known as functions of $\rho$, because of high diffusion coefficients of the charged particles and heat along the magnetic field lines.

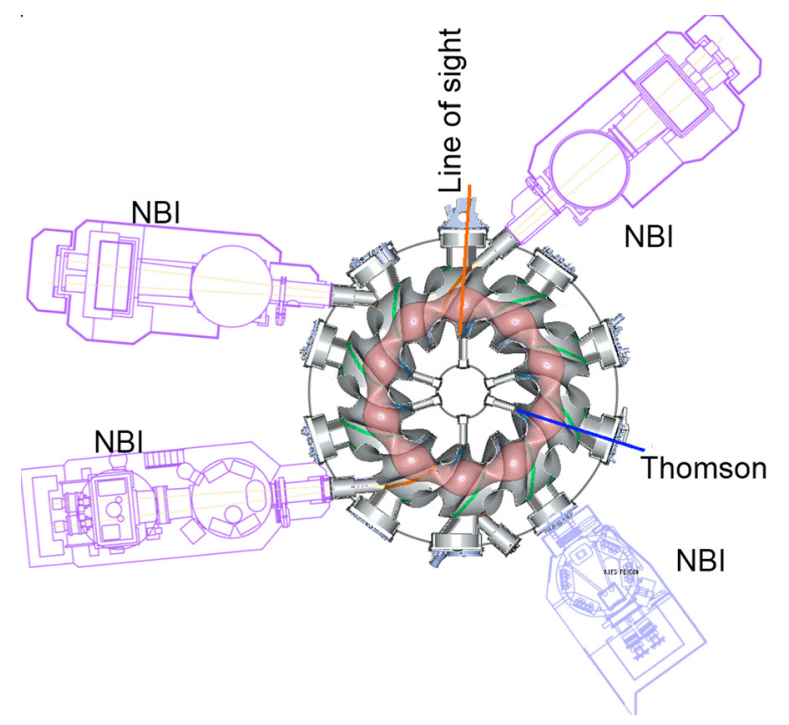

FIG. 1. A top view of LHD. Outlines of the plasma, the neutral beam injectors for the plasma heating, the laser path of the Thomson scattering method, and the LOS for the optical emission measurement are shown.

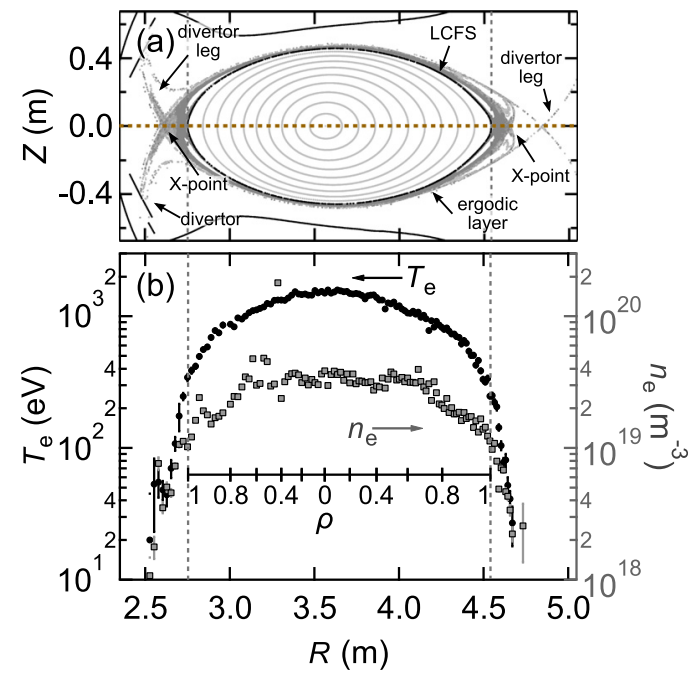

FIG. 2. (a) A Poloidal cross section of LHD for the Thomson scattering measurement. The LCFS is indicated by a black ellipsoidal curve. The gray ellipsoidal curves inside the LCFS show the magnetic flux surfaces. The gray regions outside the LCFS indicate the ergodic layer and the divertor legs. The laser path is shown by a horizontal dotted line. (b): $T_{\mathrm{e}}$ and $n_{\mathrm{e}}$ profiles measured at $t=3.17 \mathrm{~s}$. The normalized minor radius, $\rho$, along the laser path is indicated by the middle axis. The positions of the LCFS along the path are indicated with the vertical dotted lines.

The plasma parameters outside the LCFS, on the other hand, have toroidally/poloidally asymmetric spatial distributions caused by the ergodic layer and the divertor legs.

In Figure 3(a), we show LOSs for the emission measurement by horizontal lines. The LOS1 sees the divertor leg plasmas and the outmost region of the ergodic layer. The LOS2 sees the confined region as well as the ergodic layer and the divertor leg plasmas. For observations of the Zeeman

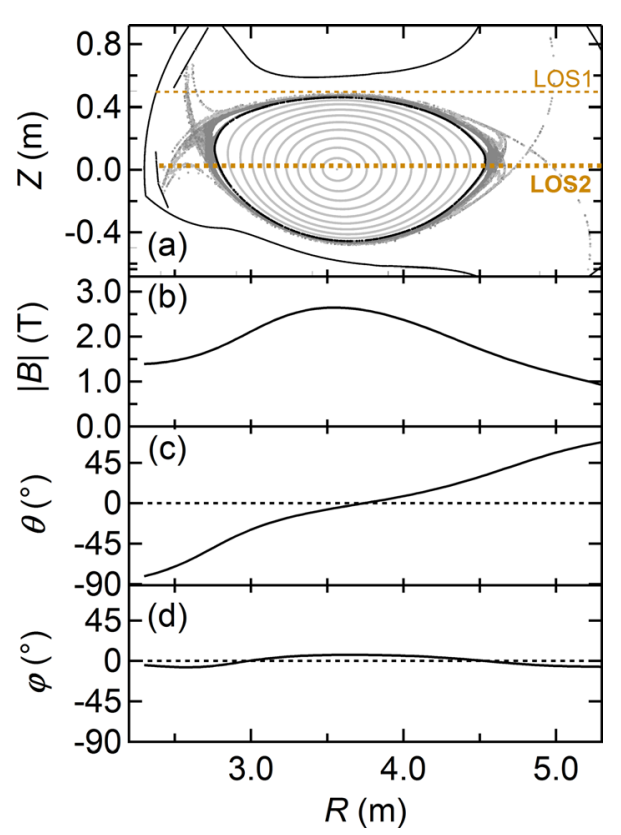

FIG. 3. (a) A poloidal cross section of LHD and the LOS for the emission measurement. The magnetic field distribution along the $\operatorname{LOS} 2(\mathrm{Z}=0.026 \mathrm{~m})$ in (a) is shown in (b), (c), and (d); (b) Magnetic field strength, $|B|$, (c) The pitch angle, $\theta$, of the magnetic field from the horizontal plane of LHD, and (d) The yaw angle, $\varphi$, between the projection of the magnetic field vector on the horizontal plane and the perpendicular direction to the LOS. 
(a)

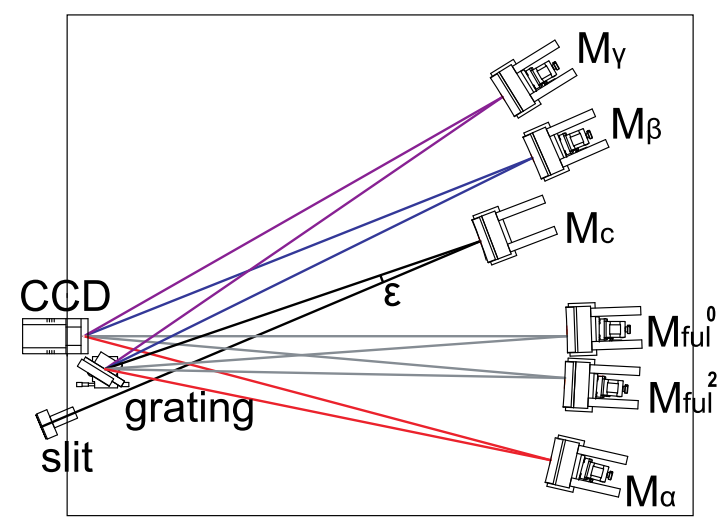

(b)

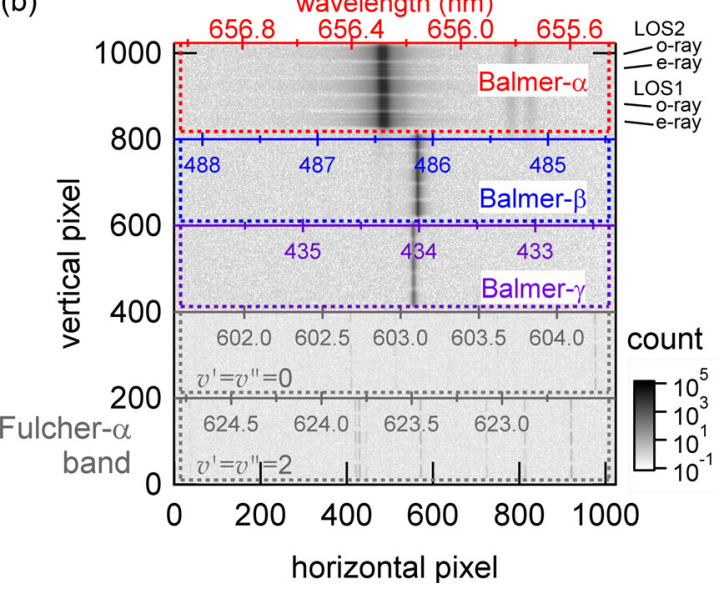

FIG. 4. (a) A schematic illustration of the MH spectrometer. (b) A two-dimensional image on the photoelectric surface observed for a low-pressure hydrogen discharge tube (Electro-Technic, SP200) with five optical fiber inputs.

splits of the emission lines, we adopt polarization separation optics (PSOs), which consist of a Glan-Thompson prism and two focusing lenses. ${ }^{7}$ The PSO are located in the outboard side of LHD and the distance between the PSO and the plasma is roughly $5 \mathrm{~m}$. Two orthogonal polarization components of the plasma emission are separately focused on the edges of two optical fibers (core diameter: $400 \mu \mathrm{m}$ ).

Figures 3(b)-3(d) shows the magnetic field strength, $|B|$, the pitch angle, $\theta$, and the yaw angle, $\varphi$, of the magnetic field lines along LOS2, respectively. The definitions of the angles are described in Ref. 7 The direction of the magnetic field is nearly perpendicular to the LOS, i.e., $\varphi \sim 0^{\circ}$, and the pitch angle at the inner ergodic layer, $\theta_{\text {in }}$, and that at the outer ergodic layer, $\theta_{\text {out }}$, are nearly perpendicular to each other, i.e., $\theta_{\text {out }}-\theta_{\text {in }} \sim 90^{\circ}$. We set the extraordinary polarization direction of the PSO to be the corresponding $\theta_{\text {in }}$ for each LOS.

The emissions are transferred by the four optical fibers with a length of $50 \mathrm{~m}$ to the entrance slit of the MH spectrometer. ${ }^{16,17}$ A schematic illustration of the spectrometer is shown in Figure 4(a). The edges of the optical fibers line along the slit with a pitch of $500 \mu \mathrm{m}$. The light introduced into the $\mathrm{MH}$ spectrometer through the slit is collimated by a concave mirror $\left(\mathrm{M}_{\mathrm{c}}\right.$ : diameter: $108 \mathrm{~mm}$ and focal length: $1143 \mathrm{~mm}$ ) and is incident on a diffraction grating (groove density: 2400 grooves $/ \mathrm{mm}$ and $104 \times 104 \mathrm{~mm}$ ). The diffracted light beams are focused on the photoelectric surface of a charge coupled device (CCD: Andor, DV435-BV, $1024 \times 1024$ pixels, $13 \times 13 \mu \mathrm{m}^{2} /$ pixel) by five focusing mirrors $\left(\mathbf{M}_{\alpha}, \mathbf{M}_{\beta}, \mathbf{M}_{\gamma}, \mathbf{M}_{\text {ful }}^{0}\right.$, and $\mathbf{M}_{\text {ful }}^{2}$, diameter: $108 \mathrm{~mm}$ and focal length: $1143 \mathrm{~mm}$ ), which are located in the direction corresponding to the wavelengths of the Balmer- $\alpha,-\beta$, and $-\gamma$ lines and the $Q$ branches of the Fulcher- $\alpha$ band with $v^{\prime}=v^{\prime \prime}=0$ and 2 transitions, where $v^{\prime}$ and $v^{\prime \prime}$ are the vibronic quantum numbers of the initial and final states, respectively.

For the purpose of explaining the measurement, we show the two-dimensional image on the photo electric surface of the CCD in Figure 4(b) for a low-pressure hydrogen discharge tube (Electro-Technic, SP200). The horizontal and vertical directions are parallel to the dispersion direction and the entrance slit, respectively. The spectra of the five emission lines are measured on the different regions of the photo- electric surface, which are indicated by colored squares in Figure 4(b). The corresponding wavelengths are indicated in the horizontal axes in Figure 4(b). Since we use five fiber inputs, five images align vertically in each region. For the LHD plasma measurement, we use upper two and lower two optical fibers for the measurement. The relation between the four images and the LOSs and their polarizations are indicated in the upper right of Figure 4(b).

The instrumental functions of the spectrometer are well approximated by a single Gauss function. The instrumental widths for the Balmer- $\alpha,-\beta$, and $-\gamma$ lines and the $Q 1$ lines in the two vibronic transitions are $8,9,10,8$, and $7 \mathrm{pm}$, respectively, at the slit width of $20 \mu \mathrm{m}$.

\section{RESULTS}

\section{A. Experiment}

In Figure 5(a), we show the temporal development of NBI heating power. A hydrogen discharge in LHD is started at $t=1.3 \mathrm{~s}$. The heating power decreases at $t=3.3 \mathrm{~s}$ and the

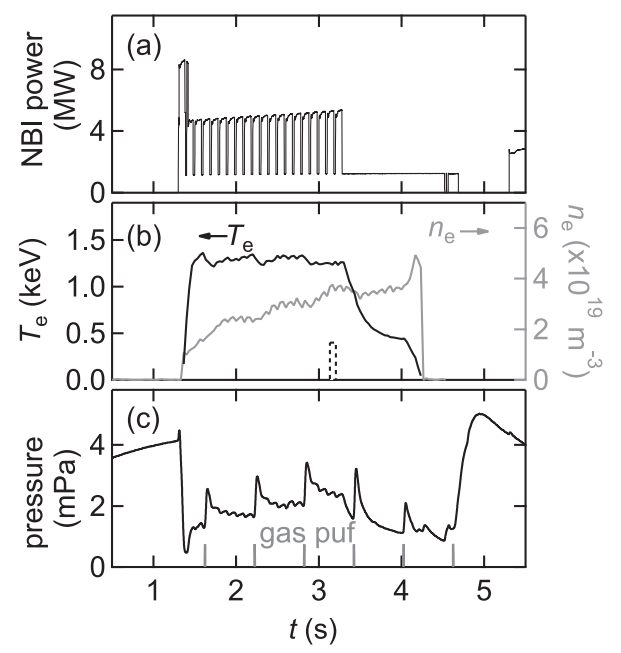

FIG. 5. Time developments of (a): the heating power of the NBI, (b): $T_{\mathrm{e}}$ and $n_{\mathrm{e}}$ at the plasma axis, and (c): neutral pressure around the inner divertor plate. The exposure time of the CCD detector is shown in (b) with a dotted square. 
heating ends at $t=4.7 \mathrm{~s}$. In Figure 5(b), the temporal developments of the electron temperature and density on the plasma axis measured by the Thomson scattering method are shown. In the discharge, repetitive hydrogen gas puffs are made. The times are shown in Figure 5(c) by gray vertical bars. According to the gas puffs, the density increases. The temperature drops after the heating power decrease. Figure 5(c) shows the time development of the neutral gas pressures around the inner divertor region measured by an ionization gauge. The pressure decreases to $1 \mathrm{mPa}$ when the plasma is generated and increases up to $3 \mathrm{mPa}$ according to the gas puffs.

We observe the emission during $t=3.14 \sim 3.21 \mathrm{~s}$. The exposure time is shown by a dotted square in Figure 5(b). Figures $6(\mathrm{a}-1)-6(\mathrm{c}-1)$ show the Balmer- $\alpha,-\beta$, and $-\gamma$ spectra, respectively, measured for LOS1. The spectra measured for LOS2 are shown in Figures 6(a-2)-6(c-2), respectively. The spectra with the ordinary polarization (o-ray) and those with the extraordinary polarization (e-ray) are shown in the upper and the lower parts of the figures, respectively. The instrumental widths for these lines are indicated by the intervals of the vertical bars in the figure.

Polarization dependences of the line shapes due to the Zeeman effect are seen in all the atomic lines. The line broadenings due to the Doppler effect are also seen in the atomic spectra. The spectra observed for LOS1 show nearly symmetric profiles while those for LOS2 show asymmetric ones.

Figures 7(a-1) and 7(b-1) show the molecular lines in the Fulcher- $\alpha$ band with $v^{\prime}=v^{\prime \prime}=0$ and 2 vibronic transitions, respectively, measured for LOS1. These spectra meas-
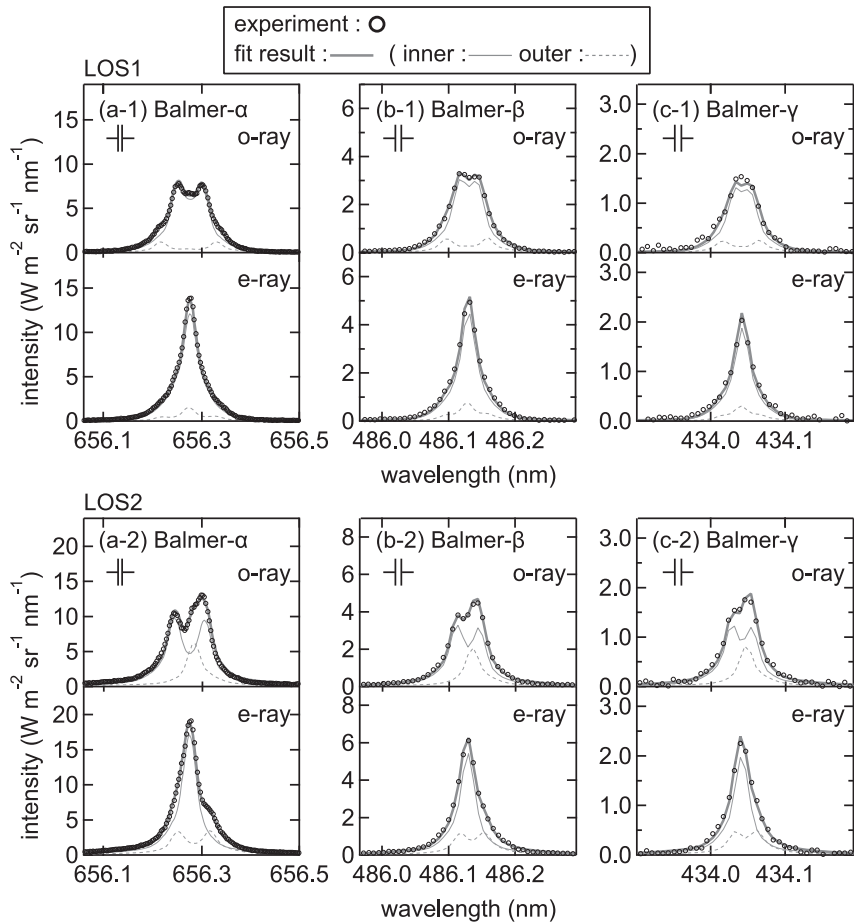

FIG. 6. (a-1): The Balmer- $\alpha$, (b-1): $-\beta$, and (c-1): $-\gamma$ spectra measured for LOS1. (a-2), (b-2), and (c-2) are those measured for LOS2. The upper and lower parts of each figure show the $o$-ray and $e$-ray components of the spectra, respectively. The instrumental widths for each wavelength region are indicated by intervals of the vertical bars. The fitting results are shown by gray bold curves. The reproduced line shapes emitted in the inner and outer emission locations are shown by gray solid and dotted curves, respectively.

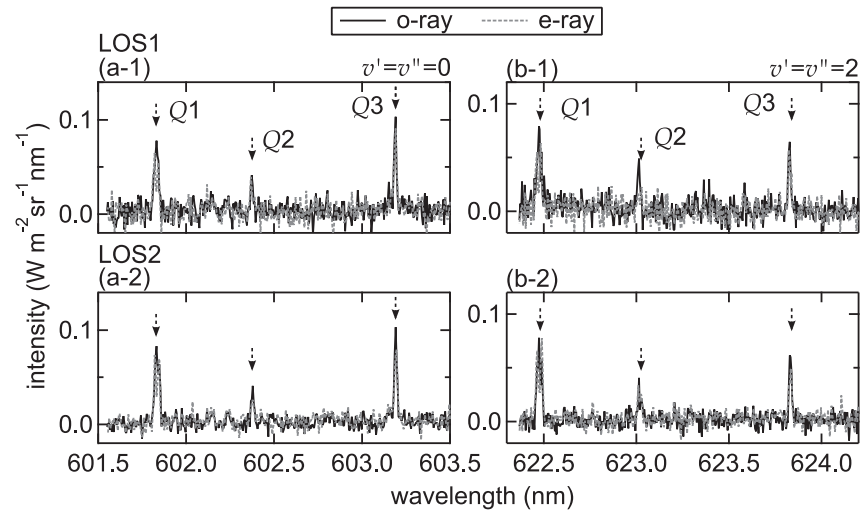

FIG. 7. The Fulcher- $\alpha$ band spectra with the vibronic transitions of (a-1 and $\mathrm{a}-2): v^{\prime}=v^{\prime \prime}=0$ and (b-1, b-2): $v^{\prime}=v^{\prime \prime}=2$. The spectra measured for LOS 1 and LOS2 are shown in (a-1 and b-1) and (a-2 and b-2), respectively. The centers of the emission lines are indicated by vertical arrows.

ured for LOS2 are shown in Figures $7(\mathrm{a}-2)$ and $7(\mathrm{~b}-2)$, respectively. The resonance wavelengths of the $Q 1, Q 2$, and Q3 lines under no magnetic field are indicated by vertical arrows.

The detail shapes of the $Q 1$ lines shown in Figures 7(a-1)-7(b-2) are shown in Figures 8(a-1)-8(b-2), respectively. The instrumental widths are indicated by intervals of

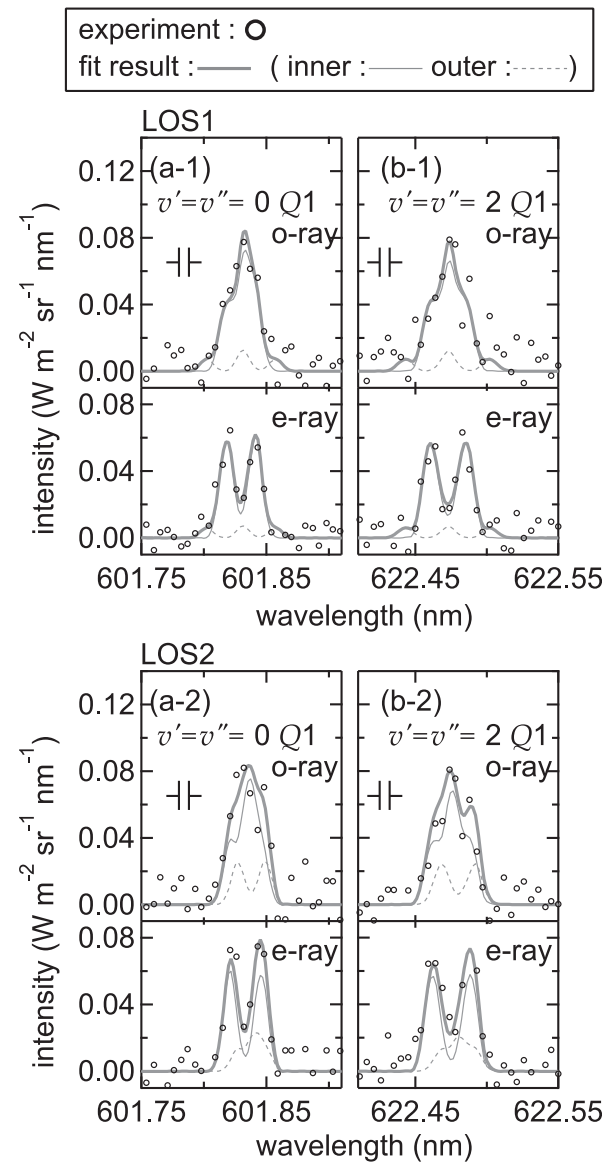

FIG. 8. The profiles of the $Q 1$ lines in the Fulcher- $\alpha$ band measured for (a-1 and $b-1)$ : LOS1 and (a-2 and b-2): LOS2. The upper and lower parts of each figure show the $o$-ray and $e$-ray components of the spectra, respectively. The instrumental widths for each wavelength region are indicated by intervals of the vertical bars. The fitting results are shown by gray bold curves. The reproduced line shapes in the inner and outer emission locations are shown by gray solid and dotted curves, respectively. 
vertical bars. The splits and the polarization dependences are also detected for the molecular lines. The Doppler broadenings of the molecular spectra are unclear in contrast with the large Doppler broadening of the Balmer series. The molecular lines measured for both the LOSs show nearly symmetric profiles. It is noted that the polarization dependences of the other rotational lines, $Q 2$ and $Q 3$ lines, are unclear because of their smaller Zeeman split ${ }^{14}$ and also their lower signalto-noise ratios due to their smaller intensities than those of the $Q 1$ line.

The $T_{\mathrm{e}}$ and $n_{\mathrm{e}}$ profiles shown in Figure 2(b) are measured for this discharge at $t=3.17 \mathrm{~s}$. The temperature and density on the plasma axis are $1.5 \mathrm{keV}$ and $3 \times 10^{19} \mathrm{~m}^{-3}$, respectively, and those on the LCFS are $400 \mathrm{eV}$ and $1.2 \times 10^{19} \mathrm{~m}^{-3}$, respectively.

\section{B. Analysis of the Balmer lines}

In the previous works by Iwamae et al., ${ }^{6,7}$ Balmer- $\alpha$ spectra measured for LHD were fitted by convolutions of Zeeman, Doppler, and instrumental profiles. Because of the steep gradient of the $T_{\mathrm{e}}$ and $n_{\mathrm{e}}$ distributions around LCFS as shown in Figure 2, most of hydrogen atoms are ionized and emit light around the LCFS. They assumed that all the atoms emit light in two points along each LOS, i.e., the inner and outer locations. From the field strengths and directions there, the Zeeman profiles of both the $o$-ray and $e$-ray components of the line shapes were reconstructed.

The velocity distribution of the hydrogen atoms was approximated by a linear combination of three shifted Maxwell distributions. ${ }^{6,19}$ The three temperature components was called as "cold," "warm," and "hot" components, respectively. They attributed the origin of the multi temperature components to the various production processes of the atoms, such as dissociations, charge exchanges, or reflections at the walls. $6,19,20$ The fittings have been made with the following 18 adjustable parameters:

- the emission locations and intensities at the inner and outer sides (4 parameters),

- the average velocities and temperatures of the cold and warm components at the inner and outer locations (8 parameters),
- the fractions of the cold and warm components at the two locations (4 parameters),

- the average velocity and temperature of the hot component, which are common in the two locations (2 parameters).

In this paper, we also adopt the same assumptions described in their papers, ${ }^{6,7}$ except that the number of the adjustable parameters is reduced. The reason of the reduction is that the changes of the shifts and widths of the broader components in the two emission locations affect only on the wing profiles of the spectra, i.e., these parameters are nearly dependent on each other and their individual values are not determined by the fittings. We assume the temperatures and the fractions of the three Maxwell distributions are identical between the inner and outer emission locations. The average velocities of the warm and hot components are also assumed to be identical. Thus, the fitting is made with the following 13 adjustable parameters in this paper:

- the emission locations and the intensities at the inner and outer sides (4 parameters)

- the average velocities of the cold and warm (hot) components at the two locations (4 parameters),

- the temperatures and fractions of the cold and warm components, which are common at the two locations (4 parameters),

- the temperature of the hot component, which are common at the both locations (1 parameters).

The results of the fittings are shown in Figures 6(a-1) and $6(\mathrm{a}-2)$ by gray bold curves. The measured spectra are well reproduced. The emission locations along LOS1 and LOS2 are derived and listed in Table I. The calculated spectra emitted at the inner and the outer locations are shown in Figures 6(a-1) and 6(a-2) by thin solid and dotted curves, respectively. Since the extraordinary direction of the PSO is set parallel to $\theta_{\text {in }}$ and the inner emission locations are close to the inner ergodic layer, the $\pi$-component $(\Delta m=0$ transition) of the Zeeman spectrum from the inner emission location is dominantly observed in the $e$-ray spectra while the $\sigma$-component ( $\Delta m= \pm 1$ transition) is dominant in the $o$-ray spectra. Here, $\Delta m$ is the difference of magnetic quantum numbers of the upper and lower states of the observed

TABLE I. The estimated parameters from the fitting of the Balmer series line shapes. The positive sign of the velocity indicate the direction toward the PSO.

\begin{tabular}{|c|c|c|c|c|c|}
\hline & & \multicolumn{2}{|c|}{ LOS1 } & \multicolumn{2}{|c|}{ LOS2 } \\
\hline & & Inner & Outer & Inner & Outer \\
\hline \multicolumn{2}{|l|}{ Emission location of atoms (m) } & 2.55 & 3.29 & 2.63 & 4.55 \\
\hline Temperatures (eV) & Cold & \multicolumn{2}{|c|}{$1.0(0.33)$} & \multicolumn{2}{|c|}{$1.16(0.36)$} \\
\hline \multirow{2}{*}{ Fractions are shown in parenthesis } & Warm & \multicolumn{2}{|c|}{$3.6(0.55)$} & \multicolumn{2}{|c|}{$4.00(0.40)$} \\
\hline & Hot & \multicolumn{2}{|c|}{$13.5(0.12)$} & \multicolumn{2}{|c|}{$19.3(0.24)$} \\
\hline \multirow[t]{2}{*}{ Velocities (km/s) } & Cold & 1.3 & 2.7 & 1.67 & -2.02 \\
\hline & Warm/Hot & 2.6 & 5.4 & 5.6 & -6.7 \\
\hline \multirow[t]{3}{*}{ Intensity $\left(\mathrm{W} \mathrm{m}^{-2} \mathrm{sr}^{-1}\right)$} & $I_{\mathrm{H} \alpha}$ & 1.63 & 0.29 & 2.24 & 0.85 \\
\hline & $I_{\mathrm{H} \beta}$ & 0.426 & 0.101 & 0.522 & 0.210 \\
\hline & $I_{\mathrm{H} \gamma}$ & 0.117 & 0.027 & 0.174 & 0.075 \\
\hline \multirow[t]{2}{*}{ Intensity ratio } & $I_{\mathrm{H} \beta} / I_{\mathrm{H} \alpha}$ & 0.26 & 0.35 & 0.23 & 0.25 \\
\hline & $I_{\mathrm{H} \gamma} / I_{\mathrm{H} \alpha}$ & 0.071 & 0.094 & 0.077 & 0.089 \\
\hline
\end{tabular}


transition. The emission intensities at the inner and outer locations, the temperatures, and the average velocities of the three Maxwell distributions and their fractions are also shown in Table I.

In Figure 9(a), the estimated emission locations and intensities of the Balmer- $\alpha$ line are shown by centers and areas of the black circles, respectively. All the atomic emission locations are estimated to be outside the LCFS. For LOS1, the inner and outer emission locations are estimated to be in the divertor leg and in the ergodic layer, respectively. For LOS2, the inner location is derived to be between the inner divertor leg and ergodic layer, while the outer one is derived to be in the ergodic layer. The intense Balmer- $\alpha$ emissions can be seen near the inner region for both LOSs. These results are consistent with the previous works. ${ }^{7}$

Although the Balmer- $\beta$ and $-\gamma$ line shapes also show splits and polarization dependences by the Zeeman effect, their magnitudes are smaller than those of the Balmer- $\alpha$ lines. It makes it difficult to separate the spectra into the two components emitted at the inner and outer locations and to derive the parameters from the fittings. We assume that these atomic lines are emitted at the same locations to those of the Balmer- $\alpha$ line. The same velocity distributions for $n=3,4$, and 5 atoms, which are the upper states of the Balmer- $\alpha,-\beta$,

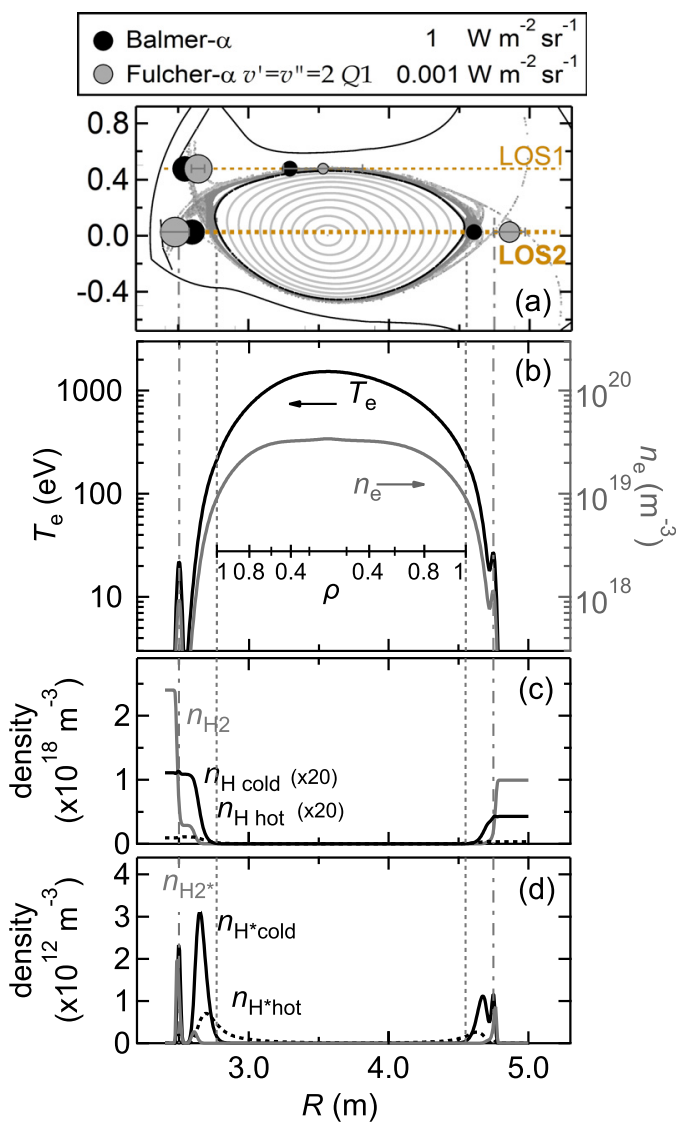

FIG. 9. (a): The emission locations and intensities of the Balmer- $\alpha$ lines are indicated by centers and areas of the black circles, respectively, and those of the $Q 1$ lines in the Fulcher- $\alpha$ band $v^{\prime}=v^{\prime \prime}=2$ transition are indicated by gray circles. (b): The assumed $T_{\mathrm{e}}$ and $n_{\mathrm{e}}$ profiles along LOS2. (c): The simulated density distributions of the hydrogen molecules and cold/hot atoms by the one-dimensional model. (d): The simulated density distributions of the excited hydrogen molecules and cold/hot atoms. and $-\gamma$ lines, respectively, are also assumed, where $n$ is the principal quantum number. The instrumental profiles are approximated by a single Gauss function also for the Balmer- $\beta$ and $-\gamma$ line shape analyses. Under the assumptions, we fit the Balmer- $\beta$ and $-\gamma$ lines with convolutions of the Zeeman, Doppler, and instrumental profiles. We adopt two adjustable parameters for the Balmer- $\beta$ line analysis, which are the emission intensities at the inner and outer emission locations. The Balmer- $\gamma$ line profile is also fitted with two adjustable parameters, which are the Bamer- $\gamma$ intensities at the inner and outer emission locations.

The fitted results of the Balmer $\beta$ and $-\gamma$ line shapes are shown by gray bold curves in Figures 6(b-1), 6(b-2), 6(c-1) and 6(c-2), respectively. The fitted results show an agreement with the measured spectra. The emission intensity ratios between the Balmer- $\alpha,-\beta$, and $-\gamma$ lines at the inner and outer locations are evaluated from the derived emission intensities there. The ratios are listed in Table I. It is noted that the uncertainties of the ratios are evaluated to be $\sim 10 \%$ for the both wavelength regions, which mainly depend on the accuracy of the sensitivity calibration of the system.

\section{Analysis of the Fulcher- $\alpha$ band}

The spectral profiles of the $Q 1$ lines in the Fulcher- $\alpha$ band shown in Figure 8 are fitted with a convolution of Zeeman, Doppler, and instrumental profiles in a similar manner to the Balmer- $\alpha$ line analysis.

We assume the emissions are localized at the inner and outer sides of the confined region on each LOS. From the assumed emission locations, the Zeeman profiles of both the $o$-ray and $e$-ray components are reconstructed. ${ }^{21}$ For calculating the Doppler profiles, we assume that the velocity distribution of the molecules is a single shifted Maxwell distribution. The translational temperature of the molecules is assumed to be identical to the rotational temperature, which is described later. The instrumental profiles are approximated by a Gauss function with the known widths.

Because of relatively small signal to noise ratios of the measured molecular lines, we assume identical emission locations for the $Q 1$ lines in the two vibronic transitions and fit them simultaneously. An identical intensity ratio of the two $Q 1$ lines is also assumed between the inner and outer emission locations. Thus, the fitting is made with the following 7 adjustable parameters:

- the emission locations and intensities of the molecules at the inner and outer sides (4 parameters),

- the average velocities of the shifted Maxwell distributions at the two locations (2 parameters),

- the common intensity ratios of the two $Q 1$ lines (1 parameters).

The fitted results of the measured spectra for LOS1 and LOS2 are shown in Figures 8(a-1), 8(b-1), 8(a-2), and 8(b-2), respectively, by gray bold curves. The emission locations are determined to be $2.64 \pm 0.05 \mathrm{~m}$ and $3.5 \pm 0.3 \mathrm{~m}$ for the inner and outer locations along LOS1, respectively. The errors are estimated from the fitting procedure. The other parameters derived from the fitting are listed in Table II. The calculated 
TABLE II. The estimated parameters from the fitting of the line shapes of the $Q 1$ lines in the Fulcher- $\alpha$ band.

\begin{tabular}{|c|c|c|c|c|c|}
\hline & & \multicolumn{2}{|c|}{ LOS1 } & \multicolumn{2}{|c|}{ LOS2 } \\
\hline & & Inner & Outer & Inner & Outer \\
\hline Emission location of molecules ( $\mathrm{m}$ & & $2.64 \pm 0.05$ & $3.5 \pm 0.3$ & $2.48 \pm 0.10$ & $4.86 \pm 0.11$ \\
\hline Rotational temperature $(\mathrm{eV})$ & & \multicolumn{2}{|c|}{$0.040 \pm 0.002$} & \multicolumn{2}{|c|}{$0.037 \pm 0.004$} \\
\hline Velocities $(\mathrm{km} / \mathrm{s})$ & & $1.7 \pm 0.3$ & $\ldots$ & $0.0 \pm 0.2$ & $-2.2 \pm 0.7$ \\
\hline Intensity $\left(\times 10^{-3} \mathrm{~W} \mathrm{~m}^{-2} \mathrm{sr}^{-1}\right)$ & $\begin{array}{l}I_{Q 1}^{v^{v^{\prime}}=2} \\
I_{Q 1}{ }^{v^{\prime}}=0\end{array}$ & $\begin{array}{c}3.6 \pm 0.2 \\
(3.7)\end{array}$ & $\begin{array}{c}0.7 \pm 0.3 \\
(0.8)\end{array}$ & $\begin{array}{c}3.4 \pm 0.3 \\
(3.6)\end{array}$ & $\begin{array}{c}1.3 \pm 0.1 \\
(1.3)\end{array}$ \\
\hline Intensity ratio & $I_{Q 1} v^{\prime^{\prime}}=0 / I_{Q 1} v^{v^{\prime}}=2$ & \multicolumn{2}{|c|}{$1.04 \pm 0.08$} & \multicolumn{2}{|c|}{$1.05 \pm 0.06$} \\
\hline
\end{tabular}

spectra emitted from the inner and outer locations are shown by thin solid and dotted curves, respectively, in Figure 8.

The estimated emission locations and intensities of the molecular lines are shown in Figure 9 by a centers and areas of gray circles. It is noted that since the emission intensities of the molecular lines are roughly three orders of magnitude smaller than those of the Balmer- $\alpha$ lines, the scales of the areas are different between these emission lines. The emission locations of the molecules are also estimated to be outside the LCFS. The molecular emission locations along LOS2 are derived to be in the divertor leg plasmas within the error bars, which are farer from the LCFS than the atomic emission locations.

Lavrov et al. have proposed a method to estimate the rotational temperature of hydrogen molecules from emission intensities of several lines in the Fulcher- $\alpha$ band. ${ }^{14}$ They have assumed that the electron impact rotational excitation probability from the $X^{1} \Sigma_{\mathrm{g}}{ }^{+}$to $d^{3} \Pi_{\mathrm{u}}{ }^{-}$state is dominated by the $\Delta N=0$ transition. Small dependences of the upper state population on the electron density have been reported. ${ }^{22}$ Although the Lavrov's model neglects such dependences, we adopt their model for the sake of simplicity. Under the Lavrov's assumption and the Boltzmann rotational distributions in both the electronic states, the following relation is satisfied:

$$
T_{\text {rot }, X}=\left(B_{X} / B_{d}\right) T_{\text {rot }, d},
$$

where $T_{\text {rot }, X}, T_{\text {rot }, d}, B_{X}$, and $B_{d}$ are the rotational temperatures and rotational energy constants of the $X^{1} \Sigma_{\mathrm{g}}{ }^{+}$and $d^{3} \Pi{ }_{\mathrm{u}}{ }^{-}$ states, respectively. $B_{\mathrm{X}}$ has been investigated to be $59.322 \mathrm{~cm}^{-1}$ by Tobias et al. ${ }^{23} B_{\mathrm{d}}$ for $v^{\prime}=0$ and 2 states are known to be 29.592 and $28.047 \mathrm{~cm}^{-1}$, respectively. ${ }^{24}$

Since the small Zeeman splits of the $Q 2$ and $Q 3$ lines make it difficult to separate these emission intensities into the inner and outer location components as the $Q 1$ line analysis described above, we assume the rotational temperatures are the same between the two locations, i.e., the emission intensity ratios of these lines are assumed to be same in the two locations. From the line integrated emission intensities shown in Figure 7, we estimate the populations in the upper states, i.e., the populations in the $d^{3} \Pi_{\mathrm{u}}^{-}$state with $\left(v^{\prime}\right.$, $\left.N^{\prime}\right)=(0,1),(0,2),(0,3)(2,1),(2,2)$, and $(2,3)$ levels, where $N^{\prime}$ is the rotational quantum number of the upper states. In Figure 10, we show the Boltzmann's plots for the populations in these states divided by their statistical weights against the rotational excitation energy of the ground state with the same rotational quantum numbers. We fit the population density distributions in the two vibronic states by exponential functions with a common exponent. The results are shown in Figure 10 by dotted lines. From the slope of the fitted result, we deduce the rotational temperatures to be $0.040 \pm 0.002 \mathrm{eV}$ and $0.039 \pm 0.004 \mathrm{eV}$ for LOS1 and LOS2, respectively. It is in the same order to the temperature of the vacuum chamber $(0.03 \sim 0.04 \mathrm{eV})$ and one or two orders of magnitude smaller than the kinetic energy of the hydrogen atoms.

The mean free path of the molecules is estimated to be larger than $1 \mathrm{~m}$ from the neutral pressure close to the divertor plates and the momentum transfer cross section. ${ }^{25}$ Because of the large mean free path, the rotational temperature may have a memory of the wall temperature. Such a dependence of the rotational temperature on the wall temperature has been reported for TEXTOR. ${ }^{22}$

\section{Spectral wings of the Balmer series}

The $e$-ray components of the Balmer series spectra and their fitted results, which are shown in Figure 6, are again shown in Figure 11 with logarithmic vertical axes. It is noted

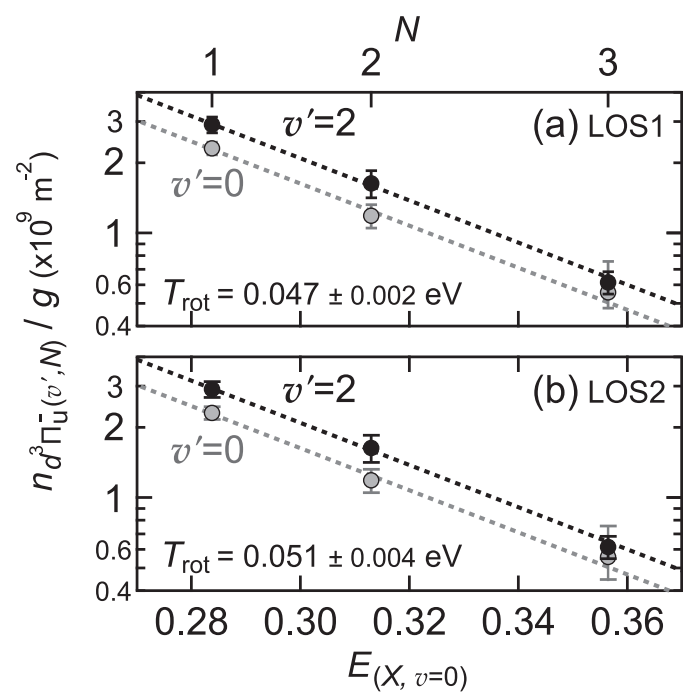

FIG. 10. The population densities in the upper states of the Fulcher- $\alpha$ band which are estimated from the rotational spectra measured for (a): LOS1 and (b): LOS2. The gray and black circles indicate the population densities in the $d^{3} \Pi_{\mathrm{u}}{ }^{-} v^{\prime}=0$ and 2 states, respectively. The horizontal axis indicates the rotational quantum number and the rotational excited energy of the $X^{1} \Sigma_{\mathrm{g}}^{+}$ $v=0$ states. The fitted results by exponential functions with a common exponent are shown by gray and black dotted lines. 

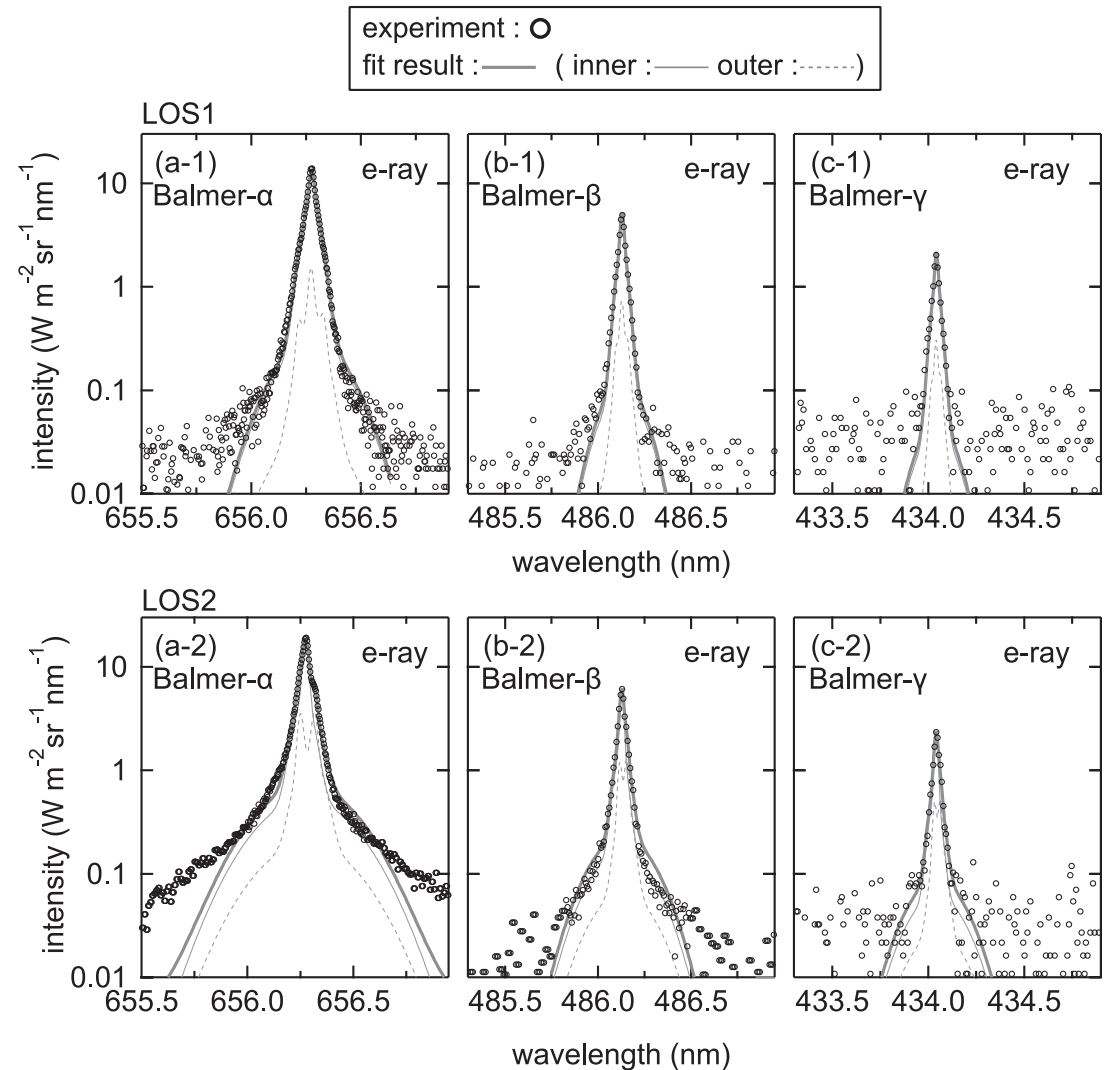

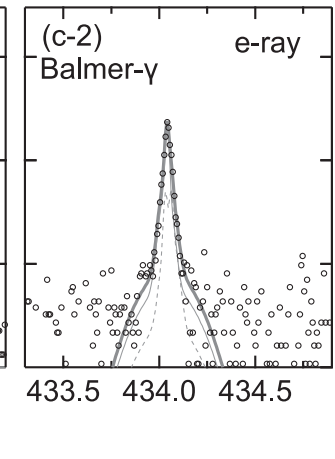

FIG. 11. The e-ray components of the Balmer series spectra shown in Figure 6 are again shown with logarithmic vertical axes. that the wavelength ranges in the figure are about three times wider than those shown in Figure 6. The intensity levels of the bremsstrahlung continuum are below the scale of Figure 11. The center parts of the spectra are well reproduced by the fittings as shown in Figure 6, however, significant far wings can be seen in the measured Balmer- $\alpha$ lines, which are not reproduced. The wings are more significant in LOS2 than in LOS1. The deviation in the line wing indicates the existence of the high velocity hydrogen atoms along $\operatorname{LOS} 2 .^{26}$

\section{ANALYSIS WITH A ONE-DIMENSIONAL HYDROGEN TRANSPORT MODEL}

\section{A. Overview of the transport model}

We consider a one-dimensional system, which consists of a plasma in the middle and walls at the both ends. We use $R$ as the spatial coordinate. The overview of the model is as follows.

We estimate the $T_{\mathrm{e}}$ and $n_{\mathrm{e}}$ profiles along LOS 2 from the results of the Thomson scattering method shown in Figure 2(b) by comparing the $\rho$ distributions. The estimated profiles are shown in Figure 9(b). The vertical dotted lines in Figure 9 indicate the positions of the LCFS. Outside the LCFS, we extrapolate the profiles with 2 nd order polynomials in the semi-logarithmic scale. Since no experimental results are available for the parameter profiles in the divertor legs, we assume them to be Gauss functions with the width of $5 \mathrm{~cm}$ as shown in Figure 9(b). The peak values are assumed to be one tenth of those on the LCFS. ${ }^{27}$ The profiles of proton density, $n_{\mathrm{p}}$, and proton temperature, $T_{\mathrm{p}}$, are assumed to be identical to the $n_{\mathrm{e}}$ and $T_{\mathrm{e}}$ profiles, respectively.

The molecular density profile is calculated under the following assumptions: Molecules are constantly desorbed from the walls and travel toward the plasma. Since the mean free path of hydrogen molecules outside the plasma is larger than $1 \mathrm{~m}$, we assume velocity of molecules do not change during the transport. The molecular density drops in the plasma because of the dissociations and ionizations by the electron impacts. The dissociation and ionization rate coefficients at each location are calculated from the collisional-radiative models and the given $T_{\mathrm{e}}$ and $n_{\mathrm{e}}$ there. In Figure 12(a), we show examples of the $T_{\mathrm{e}}$ and $n_{\mathrm{e}}$ dependences of these rate coefficients.

Atomic density distribution is calculated under the following assumptions: All atoms are created from the dissociation of the molecules. The atoms travel both directions from their generation places. In the plasma, atoms are ionized by electron impacts or colliding with protons with charge exchange. Examples of the $T_{\mathrm{e}}$ and $n_{\mathrm{e}}$ dependences of these rate coefficients are shown in Figure 12(b). The velocities of atoms are assumed not to change during the transport because of their large mean free path, but an only exception is the charge exchange collisions. Once an atom experience a charge exchange collision, the resultant atomic velocity is assumed to be the same as the velocity of colliding protons, since the forward scattering is dominant in the resonant charge exchange collision. ${ }^{28}$ Multiple charge exchange collisions during the transport are considered. All the atoms reaching the walls are assumed to be absorbed.

The density distributions of excited molecules and atoms, which are proportional to the emission intensities there are calculated from the densities of the ground state molecules/atoms, $T_{\mathrm{e}}$ and $n_{\mathrm{e}}$, and their population coefficients, which are defined as the population of the upper states per unit electron density and per unit ground state molecules/ atoms. 


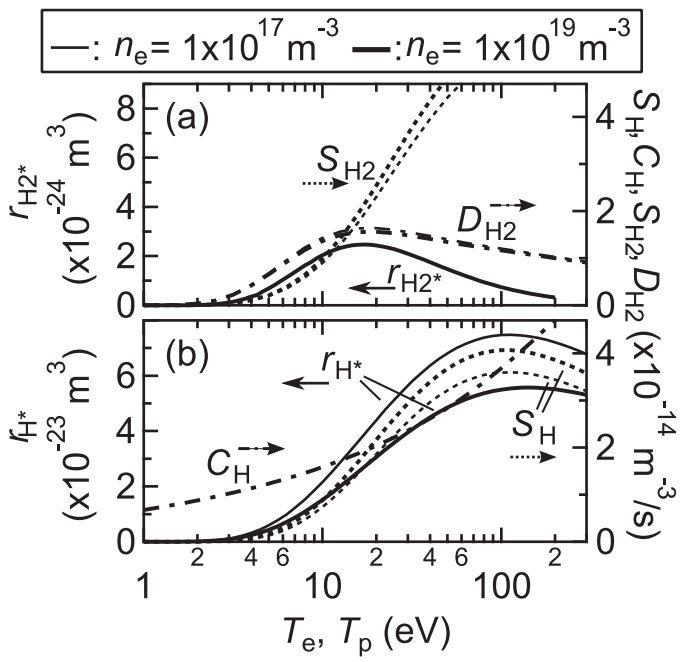

FIG. 12. (a): The population coefficients $r_{\mathrm{H} 2^{*}}$ for the $d^{3} \Pi_{\mathrm{u}}{ }^{-}\left(v^{\prime}, N^{\prime}\right)=(2,1)$ state molecule ${ }^{15}$ and the effective dissociation rate coefficients $D_{\mathrm{H} 2}$, ionization rate coefficients $S_{\mathrm{H} 2}$ for hydrogen molecules ${ }^{11}$ as a function of $T_{\mathrm{e}}$. (b): The population coefficients $r_{\mathrm{H} \alpha}$ for the $n=3$ state atom and the effective ionization rate coefficients $S_{\mathrm{H}}$ for the hydrogen atoms as a function of $T_{\mathrm{e}}{ }^{11} C_{\mathrm{H}}$ indicates a rate coefficient for the charge exchange collision with protons as a function of $T_{\mathrm{p}}$. The thick and thin curves indicate these results under $n_{\mathrm{e}}=10^{17}$ and $10^{19} \mathrm{~m}^{-3}$ cases, respectively.

\section{B. Transport calculation of hydrogen molecules}

We consider neutral hydrogen molecules desorbed at one end, $R=R_{0, \text { in }}$, and traveling toward the plasma with the positive $R$-directional velocity $v_{\mathrm{H} 2}$. The density distribution $n_{\mathrm{H} 2 \text {,in }}(R)$ is expressed as follows:

$$
\begin{aligned}
n_{\mathrm{H} 2, \mathrm{in}}(R)= & n_{\mathrm{H} 2}\left(R_{0, \text { in }}\right) \\
& \times \exp \left[-\int_{R_{0, i n}}^{R} \frac{S_{\mathrm{H} 2}\left(R^{\prime}\right)+D_{\mathrm{H} 2}\left(R^{\prime}\right)}{v_{\mathrm{H} 2}} n_{\mathrm{e}}\left(R^{\prime}\right) \mathrm{d} R^{\prime}\right],
\end{aligned}
$$

where $n_{\mathrm{H} 2 \text {,in }}\left(R_{0, \text { in }}\right)$ is the density at $R=R_{0, \text { in }}$, and $S_{\mathrm{H} 2}(R)$, and $D_{\mathrm{H} 2}(R)\left(\mathrm{m}^{3} / \mathrm{s}\right)$ are the ionization and dissociation rate coefficients, respectively, which are defined as number of molecules ionized or dissociated at $R^{\prime}$ per unit time, per unit molecular density, and per unit electron density. We adopt $1.3 \mathrm{~km} / \mathrm{s}$ as $v_{\mathrm{H} 2}$, which is the average velocity in one direction of the $0.04 \mathrm{eV}$ hydrogen molecules. The $R_{0, \text { in }}$ is set to be $2.4 \mathrm{~m}$, which is the location of the divertor plate along LOS2 shown in Figure 9(a). The density distribution of the molecules which is desorbed at another end, $R=R_{0, \text { out }}$, and travelling to the plasma with the negative $R$-directional velocity $-v_{\mathrm{H} 2}, n_{\mathrm{H} 2, \text { out }}(R)$ is described as follows:

$$
\begin{aligned}
n_{\mathrm{H} 2, \text { out }}(R)= & n_{\mathrm{H} 2}\left(R_{0, \text { out }}\right) \\
& \times \exp \left[-\int_{R_{0, \text { out }}}^{R} \frac{S_{\mathrm{H} 2}\left(R^{\prime}\right)+D_{\mathrm{H} 2}\left(R^{\prime}\right)}{-v_{\mathrm{H} 2}} n_{\mathrm{e}}\left(R^{\prime}\right) \mathrm{d} R^{\prime}\right] .
\end{aligned}
$$

We set $R_{0, \text { out }}$ to be $5.0 \mathrm{~m}$.

The resultant molecular density distribution $n_{\mathrm{H} 2}(R)$ is sum of $n_{\mathrm{H} 2 \text {,in }}(R)$ and $n_{\mathrm{H} 2 \text {,out }}(R)$. It is noted that all the calculations presented here are linearly dependent on $n_{\mathrm{H} 2}\left(R_{0, \text { in }}\right)$ and $n_{\mathrm{H} 2}\left(R_{0, \text { out }}\right)$. The determination of $n_{\mathrm{H} 2}\left(R_{0, \text { in }}\right)$ and
$n_{\mathrm{H} 2}\left(R_{0, \text { out }}\right)$ is described later. The calculated result is shown in Figure 9(c) by a gray curve. The ionization and dissociation flux of the molecules, $\Phi^{S}{ }_{\mathrm{H} 2}(R)$ and $\Phi^{D}{ }_{\mathrm{H} 2}(R)$, which are defined as the number of molecules ionized and dissociated per unit time per unit volume, respectively, are calculated as follows:

$$
\begin{aligned}
\Phi^{\mathrm{S}}{ }_{\mathrm{H} 2}(R) & =S_{\mathrm{H} 2}(R) n_{\mathrm{e}}(R) n_{\mathrm{H} 2}(R), \\
\Phi^{\mathrm{D}}{ }_{\mathrm{H} 2}(R) & =D_{\mathrm{H} 2}(R) n_{\mathrm{e}}(R) n_{\mathrm{H} 2}(R) .
\end{aligned}
$$

The density distribution in the $d^{3} \Pi_{\mathrm{u}}{ }^{-}\left(v^{\prime}, N^{\prime}\right)=(2,1)$ state, $n_{\mathrm{H} 2 *}(R)$, is expressed as follows:

$$
n_{\mathrm{H} 2^{*}}(R)=r_{\mathrm{H} 2^{*}}(R) n_{\mathrm{e}}(R) n_{\mathrm{H} 2}(R),
$$

where, $r_{\mathrm{H} 2 *}$ is the population coefficient for the $d^{3} \Pi_{\mathrm{u}}{ }^{-}\left(v^{\prime}\right.$, $\left.N^{\prime}\right)=(2,1)$ state, which is calculated with the coronal model developed by Shikama et al. ${ }^{14}$ The temperature dependence of $r_{\mathrm{H} 2 *}$ is shown in Figure 12(a). We assume the vibronic temperature, $T_{\mathrm{vib}}$, of the ground state molecules as $0.3 \mathrm{eV}$, which has been measured in TRIAM-1 $\mathrm{M}$ by Shikama et al. ${ }^{14}$ It is noted that the dependences of $T_{\mathrm{vib}}$ on $r_{\mathrm{H} 2 *}$ is small. For example, $r_{\mathrm{H}^{*}}$ in $T_{\mathrm{vib}}=0.2 \mathrm{eV}$ is roughly $15 \%$ smaller than that in $T_{\mathrm{vib}}=0.4 \mathrm{eV}$.

The result is shown in Figure 9(d) by a gray curve. The results of $n_{\mathrm{H} 2^{*}}(R), \Phi^{S}{ }_{\mathrm{H} 2}(R)$, and $\Phi^{D}{ }_{\mathrm{H} 2}(R)$ near the inner divertor leg region are shown in Figure 13(b) with a magnification. Most of the hydrogen molecules are ionized/dissociated in the divertor legs, and the dominant emission locations are also in the divertor legs. The width of the $n_{\mathrm{H} 2 *}(R)$ is about $2 \mathrm{~cm}$, which is consistent with the assumption adopted in the spectral shape analysis that emission locations are localized. The ionization and dissociation flux distributions are almost identical to the emission distribution. It is due to the similar tendency between the ionization, dissociation, and population coefficients on $T_{\mathrm{e}}$ and small dependences on $n_{\mathrm{e}}$ as shown in Figure 12(a).

\section{Transport calculation of hydrogen atoms}

In the spectral analysis of the Balmer series, the atomic velocity distribution is assumed to be a linear combination of the cold, warm, and hot components. The cold and warm components are considered to be mainly originated from desorption from the wall and dissociation from the molecules, while the hot component is due to charge exchange collision.

In the one-dimensional model, we simplify the situation that the atoms are assumed to be generated only from the dissociation and charge exchange collisions. In the model, the cold atoms are defined as the atoms, which are generated from the dissociation and they are diminished by the ionization by the electron impact and the charge exchange collisions with protons. The velocity distribution of the dissociated atoms is known to have a specific profile, which depends on dissociation paths. We assume the velocity distribution of the cold atoms to be a single Maxwell distribution with the temperature of $3 \mathrm{eV}$, which is an approximation of that dissociated from the vibronic ground state in $X^{1} \Sigma_{\mathrm{g}}^{+}$ state through the lowest dissociative state, $b^{3} \Sigma_{\mathrm{u}}^{+}$state. ${ }^{29}$ The 
atoms generated from the protons through the charge exchange collisions are grouped into the hot atoms.

Probability of the atoms, which is generated in position $R^{\prime}$ to reach the $R$ with velocity $v, P\left(R, R^{\prime}, v\right)$, decreases with the increase of the travelling distance $\left|R-R^{\prime}\right|$ by the ioniza- tion and the charge exchange collisions. We further assume the rates of the ionization and the charge exchange collisions have no dependences on the atom velocity $v . P\left(R, R^{\prime}, v\right)$ is expressed with the corresponding rate coefficients, $S_{\mathrm{H}}(R)$ and $C_{\mathrm{H}}(R)$, as follows:

$$
P\left(R, R^{\prime}, v\right)=\left\{\exp \left[-\int_{R^{\prime}}^{R} \frac{S_{\mathrm{H}}\left(R^{\prime \prime}\right)+C_{\mathrm{H}}\left(R^{\prime \prime}\right)}{v} n_{\mathrm{e}}\left(R^{\prime \prime}\right) \mathrm{d} R^{\prime \prime}\right] \quad \begin{array}{ll}
\left(\left(R-R^{\prime}\right) \times v>0\right) \\
0 & \left(\left(R-R^{\prime}\right) \times v \leq 0\right)
\end{array} .\right.
$$

It is noted that no atoms reach $R$ from $R^{\prime}$ with opposite directional velocity to $\left(R-R^{\prime}\right)$.

Number of the cold atoms, which is generated in $R^{\prime} \sim R^{\prime}$ $+\mathrm{d} R^{\prime}$ and with velocity of $v \sim v+\mathrm{d} v$ to reach $R$ per unit time, $F_{\text {cold }}\left(R, R^{\prime}, v\right) \mathrm{d} R^{\prime} \mathrm{d} v\left(\mathrm{~m}^{-2} \mathrm{~s}^{-1}\right)$, can be written with $P\left(R, R^{\prime}, v\right)$ and $\Phi^{D}{ }_{\mathrm{H} 2}(R)$ as follows:

$F_{\text {cold }}\left(R, R^{\prime}, v\right) \mathrm{d} R^{\prime} \mathrm{d} v=2 \Phi^{D}{ }_{\mathrm{H} 2}\left(R^{\prime}\right) \mathrm{d} R^{\prime} f_{\mathrm{H}}\left(v, T_{\mathrm{dis}}\right) \mathrm{d} v P\left(R, R^{\prime}, v\right)$,

where the two corresponds to the number of atoms generated via dissociation per one molecule and $f_{\mathrm{H}}\left(v, T_{\mathrm{dis}}\right)$ is the veloc-
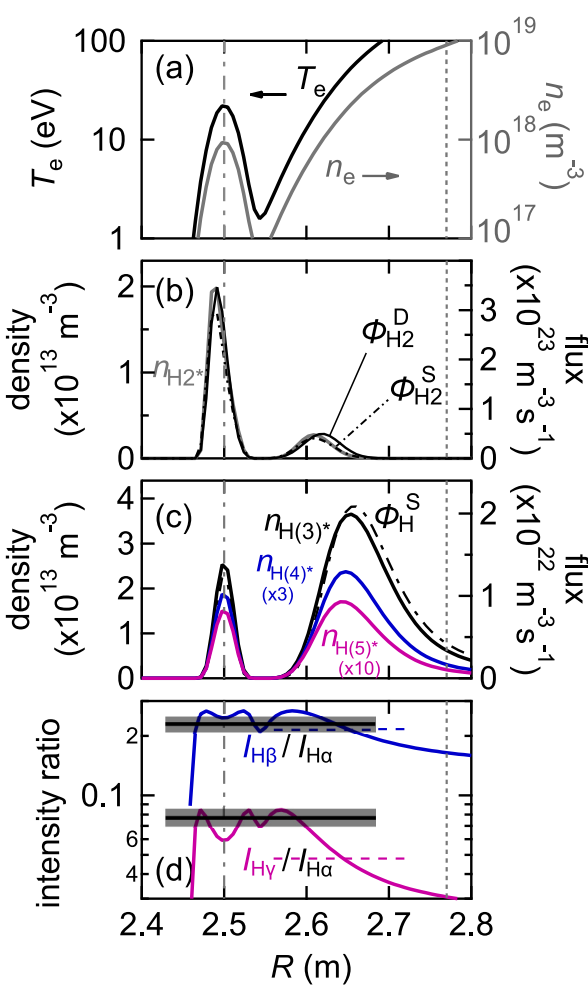

FIG. 13. (a) The assumed $T_{\mathrm{e}}$ and $n_{\mathrm{e}}$ distributions along LOS2 in $R=2.4 \sim 2.8 \mathrm{~m}$. (b): The simulated distributions of the excited molecular density (gray solid curve), the dissociation flux (black solid curve), and the ionization flux of the molecules (black dashed-dotted curve). (c): The simulated distributions of the excited atomic density (solid curve) and the ionization flux of the atoms (black dashed-dotted curve). (d): The simulated distributions of emission intensity ratios of the Balmer series lines. The ratios of the line integrated intensities over $R=2.4 \sim 2.75 \mathrm{~m}$ are shown by horizontal dotted lines. The intensity ratios estimated from the spectral analysis are indicated by horizontal lines. ity distribution of the dissociated atoms, which is assumed to be a Maxwell distribution with a temperature of $3 \mathrm{eV}$.

The density of the cold atoms having the velocity of $v \sim$ $v+\mathrm{d} v$ at the position $R, n_{\text {Hcold }}(R, v) \mathrm{d} v\left(\mathrm{~m}^{-3}\right)$, can be deduced from the integration of $F_{\text {cold }}\left(R, R^{\prime}, v\right) \mathrm{d} v$ over $R^{\prime}$ and division by $v$, as follows:

$$
n_{\text {Hcold }}(R, v) \mathrm{d} v=\int_{R_{0, \text { in }}}^{R_{0, \text { out }}} F_{\text {cold }}\left(R, R^{\prime}, v\right) \mathrm{d} R^{\prime} \mathrm{d} v / v \quad(v \neq 0) .
$$

The density of the cold atoms at the position $R, n_{\mathrm{Hcold}}(R)$ $\left(\mathrm{m}^{-3}\right)$ is derived from $n_{\text {Hcold }}(R, v)$ by integrating over $v$,

$$
n_{\mathrm{Hcold}}(R)=\int_{-\infty}^{\infty} n_{\mathrm{Hcold}}(R, v) \mathrm{d} v .
$$

The calculated $n_{\text {Hcold }}(R)$ is shown in Figure 9(c) with a solid curve. The nonzero density is calculated outside the plasma $\left(R<R_{0, \text { in }}\right)$ while the dominant generation place is $R \sim 2.65 \mathrm{~m}$ as shown in Figure 13(b), since half of the dissociated atoms travel with negative velocity.

As for the hot atoms, velocity changes of the multiple charge exchange collisions make the calculation complex. We improve the calculation method reported by Tamor. ${ }^{30}$ The details of the calculation method are described in Appendix. The derived density distribution of the hot atoms, $n_{\mathrm{H} \text { hot }}(R)$, is shown in Figure 9(c) by a dotted curve. The spatial and velocity distributions of the cold and hot atoms in the $n=3$ state, $n_{\mathrm{H}^{*} \text { cold }}(v, R)$ and $n_{\mathrm{H} * \text { hot }}(v, R)$, respectively, are calculated with the population coefficient for the $n=3$ atoms, $r_{\mathrm{H}^{*}}(R)$, as follows:

$$
\begin{aligned}
n_{\mathrm{H}^{*} \text { cold }}(R, v) & =r_{\mathrm{H}^{*}}(R) n_{\mathrm{e}}(R) n_{\mathrm{Hcold}}(R, v), \\
n_{\mathrm{H}^{*} \text { hot }}(R, v) & =r_{\mathrm{H}^{*}}(R) n_{\mathrm{e}}(R) n_{\mathrm{Hhot}}(R, v) .
\end{aligned}
$$

Their spatial distributions, $n_{\mathrm{H} * \text { cold }}(R)$ and $n_{\mathrm{H} * \text { hot }}(R)$, which is defined as the integrations of the spatial and velocity distributions over $v$ are shown in Figure 9(d) by black solid and dotted curves, respectively. Here, $r_{\mathrm{H}^{*}}(R)$ is calculated with the collisional-radiative model assuming the ionizing plasma. ${ }^{11}$ The $T_{\mathrm{e}}$ and $n_{\mathrm{e}}$ dependences of the coefficient are shown in Figure 12(b). In Figure 13(c), we show the ionization flux distribution, $\Phi_{\mathrm{H}}^{S}(R)=S_{\mathrm{H}}(R) n_{\mathrm{e}}(R)\left(n_{\mathrm{Hcold}}(R)+\right.$ $\left.n_{\text {Hhot }}(R)\right)$, by a dashed-dotted curve. 
Most of the hydrogen atoms pass through the divertor legs and penetrate into the ergodic layer. The dominant emission locations are in the ergodic layer, i.e., the emission locations of the atoms are simulated to be closer to the core plasma than those of the molecules. The tendency is consistent with the results of the spectral analyses. The width of the emission distribution is estimated to be about $10 \mathrm{~cm}$. The position of the emission and ionization are almost identical, which means the intense atomic emission is generated at the dominant ionization locations.

$n_{\mathrm{H}(4)^{*}}$ and $n_{\mathrm{H}(5)^{*} \text {, which are the excited atom density in }}$ the $n=4$ and 5 states, respectively, are calculated from and the population coefficients there for these states. The distributions are also shown in Figure 13(c). The intensity distributions of the Balmer series are calculated from the result. The distributions of the emission intensity ratios, which are defined as $I_{\mathrm{H} \beta}(R) / I_{\mathrm{H} \alpha}(R)=h \nu_{\mathrm{H} \beta} n_{\mathrm{H}(4)^{*}} / h \nu_{\mathrm{H} \alpha} n_{\mathrm{H}^{*}}$ and $I_{\mathrm{H} \gamma}(R) / I_{\mathrm{H} \alpha}(R)$ $=h \nu_{\mathrm{H} \gamma} n_{\mathrm{H}(5) *} / h \nu_{\mathrm{H} \alpha} n_{\mathrm{H}^{*}}$, where $h$ is Planck constant and $\nu_{\mathrm{H} \alpha}$, $\nu_{\mathrm{H} \beta}$, and $\nu_{\mathrm{H} \gamma}$ are frequencies of the Balmer- $\alpha,-\beta$, and $-\gamma$ lines, respectively, are shown in Figure 13(d). The ratios vary within the intense emission region. For example, $I_{\mathrm{H} \gamma}(R) / I_{\mathrm{H} \alpha}(R)$ varies from 0.03 to 0.06 between $R=2.6 \sim 2.75 \mathrm{~m}$. The ratios of the integrated intensities over $R$ from 2.4 to $2.75 \mathrm{~m}$, which is the inner region outside the LCFS, are 0.22 and 0.048 for $I_{\mathrm{H} \beta} / I_{\mathrm{H} \alpha}$ and $I_{\mathrm{H} \gamma} / I_{\mathrm{H} \alpha}$, respectively. The integrated intensity ratios are indicated in Figure 13(d) by horizontal dotted lines. The cross points are almost at the center of the intense location, where $n_{\mathrm{e}}$ is $\sim 2 \times 10^{18} \mathrm{~m}^{-3}$.

In Figure 14, we show the emission intensity ratios, $I_{\mathrm{H} \beta} /$ $I_{\mathrm{H} \alpha}$ and $I_{\mathrm{H} \gamma} / I_{\mathrm{H} \alpha}$, calculated with the collisional-radiative model as functions of $n_{\mathrm{e}}$ and $T_{\mathrm{e}}$. It is noted that the ratios shown in the figure are only dependent on $n_{\mathrm{e}}$ and $T_{\mathrm{e}}$, and no atom transfers or line-integrations of the emissions are considered. The intensity ratios are mainly dependent on $n_{\mathrm{e}}$ and higher intensity ratios are predicted in lower electron density. The emission intensity ratios in the inner region along LOS2 estimated from the spectral analysis are shown in Figures 13(d) and 14 with horizontal solid bars. In Figure 13(d), the errors are shown by the thickness of the bars. $I_{\mathrm{H} \beta} / I_{\mathrm{H} \alpha}$ simulated by the model is within the error while $I_{\mathrm{H} \gamma} / I_{\mathrm{H} \alpha}$ is

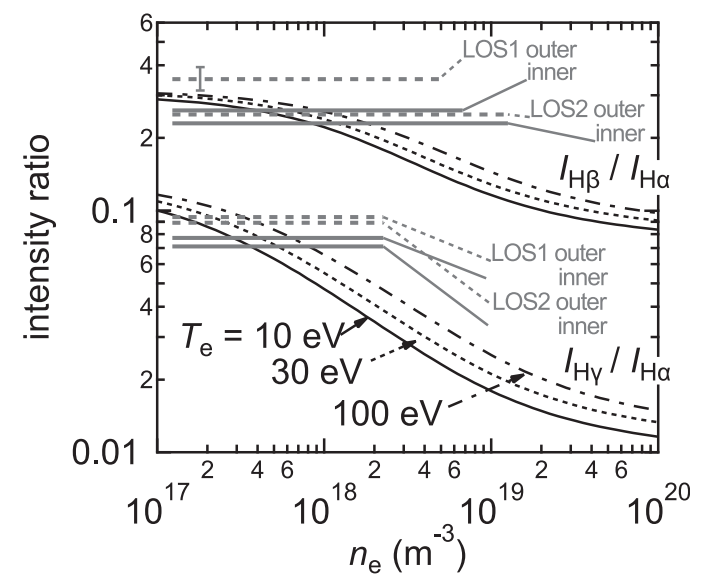

FIG. 14. Emission intensity ratios of the Balmer- $\beta$ and- $\gamma$ lines against for the Balmer- $\alpha$ line in an ionizing plasma. ${ }^{11}$ The horizontal gray lines indicate the measured intensity ratios. smaller than those of the experiment and the discrepancy is not explained by the experimental errors. $n_{\mathrm{e}}$ at the emission location corresponds to the measured intensity ratio is $\sim 5 \times 10^{17} \mathrm{~m}^{-3}$, which is four times smaller than that by the model.

The ionization location or the emission location is determined by $f_{\mathrm{H}}\left(v, T_{\mathrm{dis}}\right)$ and the integration of $S_{\mathrm{H}} n_{\mathrm{e}}$ as shown in Eqs. (6) and (7). $T_{\text {dis }}$ of $3 \mathrm{eV}$ is nearly consistent with the result of the spectral analysis. Since the electron temperature and density distributions outside the LCFS are roughly estimated in this model, we made additional calculations with the different parameter distributions outside the LCFS. However, almost same intensity ratios were calculated. The discrepancy of the ratios is not explained by the misestimation of the parameter distributions.

On the other hand, the contributions of the dissociative excitation of the molecules and molecular ions to the atomic emission intensity ratios which are not included in the above calculation have been discussed recently. ${ }^{31,32}$ The contribution of the dissociative excitation of the molecules on the Balmer series line intensity has been reported by Sawada and Fujimoto. ${ }^{10,11}$ From their work, the emission component of the dissociative excitation is estimated to be roughly $1 / 3$ of that from the ground state atoms. Since the intensity ratios will be slightly lowered by the contribution, ${ }^{11}$ the discrepancy of the intensity ratios is not explained by the process.

For the molecular ions, their transport is not considered in the model because it is affected by the three-dimensional magnetic field distribution. Additionally, the cross sections have a dependence on the vibronic temperature of the molecular ions, which is unavailable in the experiment. ${ }^{33}$ A possible reason of the discrepancy is the contribution from the molecular ions. Quantitative considerations of the processes are desired for later works.

The calculated density distributions shown in Figures 9(b) and 9(c) are again shown in Figures 15(a) and 15(b), respectively, with a semi-logarithmic scale. It can be seen that the hot atoms exist even in the core region of the plasma. The spatial and velocity distribution of the $n=3$ atoms, $n_{\mathrm{H} *}(v, R)=n_{\mathrm{H}^{*} \text { cold }}(v, R)+n_{\mathrm{H} * \text { hot }}(v, R)$, is shown in Figure 15 (c) with a color scale. It can be seen that the high velocity atoms are dominantly exist inside the LCFS.

We construct the Doppler and Zeeman profile of the Balmer- $\alpha$ line from $n_{\mathrm{H}^{*}}(v, R)$ by the line integration along $R$ axis assuming the same magnetic field distribution along LOS2. The instrumental function is also considered. The calculated $e$-ray profile of the Balmer- $\alpha$ line is shown in Figure 16 by a bold curve. In the figure, the experimental result is also shown by open circles. It is noted that $n_{\mathrm{H} 2}\left(R_{0, \text { in }}\right)$ and $n_{\mathrm{H} 2}\left(R_{0, \text { out }}\right)$ in the Eqs. (2) and (3) are calculated so that the simulated intensity reproduces the measured result.

The constructed line shape also shows exponential-like wings. In the top axis of Figure 15(a), we show the normalized radius $\rho$ along LOS 2 . The calculated line profiles which are line integrated in the region of $1<\rho, 0.8<\rho<1$, $0.4<\rho<0.8$, and $\rho<0.4$ are also shown in Figure 6 by thin solid, dotted, dashed, and dashed-dotted curves, respectively. The intense emission and narrow profile from the $1<\rho$ region are calculated. The emission intensity from the $1<\rho$ 


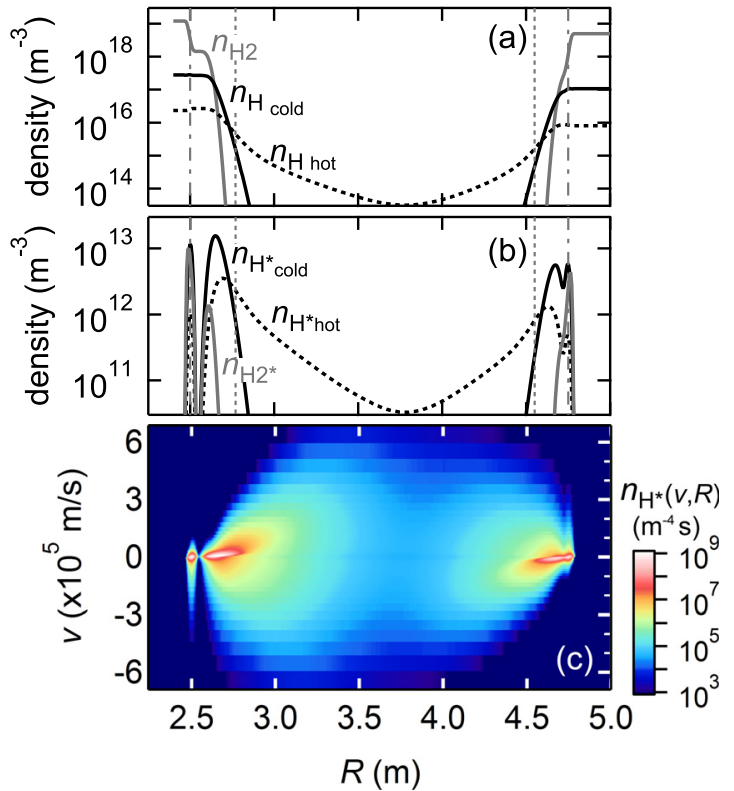

FIG. 15. The simulated density distributions of (a): the molecules, the cold atoms, and hot atoms, and (b): the excited molecules, the excited cold atoms and excited hot atoms. They are already shown but the vertical axes are logarithmic. In the top axis in (a), the normalized radius $\rho$ along LOS2 is indicated. (c): The spatial and velocity density distributions of the excited atoms in the $n=3$ state. The color scale is also logarithmic.

region dominates $86 \%$ of the entire line intensity. The weak emission and wide profile from the inside of the LCFS are calculated. The comparison indicates that the emission around the line center of the Balmer- $\alpha$ series spectra is originated from the outside of the LCFS, i.e., from cold regions, and that in the wings comes from the inside of the LCFS, i.e., from the hot regions. The indication is consistent with

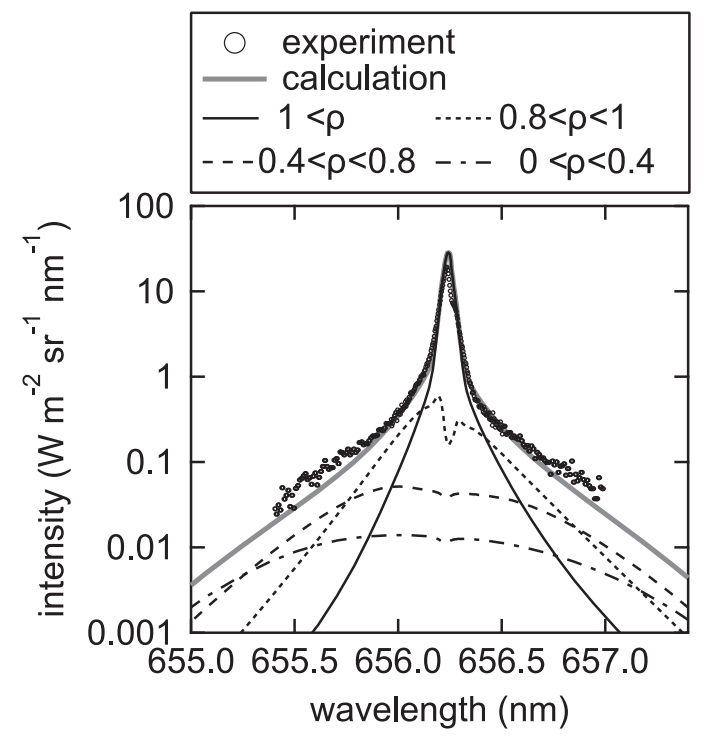

FIG. 16. The Balmer- $\alpha$ spectra measured for LOS2 in semi-logarithmic scale (open circles). The gray bold curves show the simulated $e$-ray spectra of the Balmer- $\alpha$ line by a one-dimensional model integrated over entire region. The partially integrated spectra, i.e., integrated over the regions of $1<\rho, 0.8<\rho<1,0.4<\rho<0.6$, and $0<\rho<0.4$ are shown by solid, dotted, dashed, and dashed-dotted thin curves, respectively. The large intensity and the narrow profile from the outside of the LCFS $(1<\rho)$ is calculated, while the small intensities and wide profiles are calculated inside the LCFS.

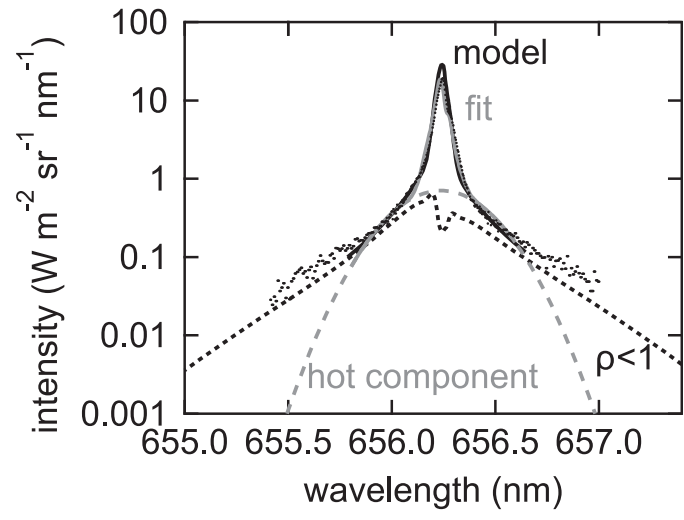

FIG. 17. A comparison of the Balmer- $\alpha$ spectra constructed by the spectral analysis and the one-dimensional model. The open circles indicate the measured spectrum for LOS2. The black solid and dotted curves indicate the constructed spectra and its hot component by the spectral analysis. The gray solid and dotted curves are the constructed spectra and $1<\rho$ component the one-dimensional model.

the small wing intensity of the Balmer- $\alpha$ spectrum measured for LOS1, which only sees lower temperature region.

Since in the center region of the atomic line profiles where the Zeeman split appears the emission intensity from the outside of the LCFS is two orders of magnitude larger than those from the inside of the LCFS, the emission locations derived from the analysis of the Zeeman split are confirmed to be not affected by the emission from the confined region.

On the other hand, the intensities derived from the spectral analysis are affected by the emission from the confined region since it includes the wing intensities. In Figure 17, we show the hot component of the constructed spectra by the spectral analysis and the $1<\rho$ component constructed by the one-dimensional model are shown. The hot component by the spectral analysis roughly indicates the $1<\rho$ component. The fraction of the hot component in the total emission intensity is 0.24 while that of the $1<\rho$ component is 0.14 .

It is noted that the emission from the inside of the LCFS itself provides no explanations about the discrepancy of the intensity ratio of the model and the experiment, since the intensity ratios of the model including inside the LCFS are 5\% smaller than those outside the LCFS, which is shown in Figure 13(d) by horizontal dotted lines, while experimental results are larger.

\section{CONCLUSION}

We observed profiles of the Balmer- $\alpha,-\beta$, and $-\gamma$ lines of hydrogen atoms and the $Q$ branches in the Fulcher- $\alpha$ band of the hydrogen molecules simultaneously with their polarization resolved for an LHD plasma. From the analysis of the line splits and the polarization dependences due to the Zeeman effect of the Balmer series lines, the emission locations and the intensities of the hydrogen atoms, and the temperature components of the atomic velocity distributions are determined. From the spectral analysis of the molecular lines, the emission locations, the intensities, and the rotational temperature of the hydrogen molecules are determined. The emission locations of the molecules are close to the divertor legs while those of atoms are closer to the LCFS, where the density and temperature are consider to be 
higher than in divertor legs. The temperatures of the hydrogen atoms are in the order of $1 \sim 20 \mathrm{eV}$, while the rotational temperature of the molecules are $0.04 \mathrm{eV}$. Addition to them, it is found that the Balmer series lines have substantial high velocity wings, which cannot be reproduced by the conventional analytical methods.

We develop a one-dimensional model to simulate the transport of the hydrogen atoms and molecules. The differences of the emission locations are reproduced by the model, in which the $0.04 \mathrm{eV}$ for molecular temperature and $3 \mathrm{eV}$ for initial atomic temperature are assumed. The high velocity tails of the hydrogen atoms are also reconstructed and are attributed to be high velocity atoms generated by charge exchange collisions inside the LCFS, while most of atoms are ionized and emit light outside the LCFS. The emission intensity ratios between the Balmer series lines by the model are not consistent to the measurement result. No clear reasons of the discrepancy are found. Additional excitation processes of atoms which are not included the model, for example, the dissociative excitations from hydrogen molecules or molecular ions, are one of the candidates.

\section{ACKNOWLEDGMENTS}

The authors are grateful to the LHD experimental group for their support. This work was supported by the National Institute for Fusion Science (Grant Nos. NIFS08KOAP020 and NIFS10KLMP003).

\section{APPENDIX: TRANSPORT CALCULATION OF HOT ATOMS INCLUDING MULTIPLE CHARGE EXCHANGE COLLISIONS}

Calculation of the spatial and velocity density distribution of the hot atoms in this paper is based on the method in Ref. 30, which is summarized here.
Number of the hot atoms generated in $R^{\prime} \sim R^{\prime}+\mathrm{d} R^{\prime}$ and travels to $R$ with velocity of $v \sim v+\mathrm{d} v$ per unit time, $F_{\text {hot }}\left(R, R^{\prime}, v\right) \mathrm{d} R^{\prime} \mathrm{d} v\left(\mathrm{~m}^{-2} \mathrm{~s}^{-1}\right)$, can be written with the number of created atoms by charge exchange at $R^{\prime}$ per unit time and per unit volume, $\Phi_{\mathrm{CX}}\left(R^{\prime}\right)\left(\mathrm{m}^{-3} \mathrm{~s}^{-1}\right)$, as the following form, which is similar to Eq. (7):

$F_{\text {hot }}\left(R, R^{\prime}, v\right) \mathrm{d} R^{\prime} \mathrm{d} v=\Phi_{\mathrm{CX}}\left(R^{\prime}\right) \mathrm{d} R^{\prime} f_{\mathrm{H}}\left(v, T_{\mathrm{p}}\left(R^{\prime}\right)\right) \mathrm{d} v P\left(R, R^{\prime}, v\right)$,

where $f_{\mathrm{H}}\left(v, T_{\mathrm{p}}\left(R^{\prime}\right)\right)$ is the velocity distribution of the created atoms by charge exchange at $R^{\prime}$, which is now assumed to be a single Maxwell distribution with the proton temperature there. Since $\Phi_{\mathrm{CX}}(R)$ should be equal to the number of atoms diminished by charge exchange collisions at $R^{\prime}, \Phi_{\mathrm{CX}}(R)$, which is an unknown distribution to be obtained, are written with the following form:

$$
\begin{aligned}
\Phi_{\mathrm{CX}}(R)= & C_{\mathrm{H}}(R) \int_{R_{0, \text { in }}}^{R_{0, \text { out }}} \mathrm{d} R^{\prime} \int \mathrm{d} v\left[F_{\text {cold }}\left(R, R^{\prime}, v\right)\right. \\
& \left.+F_{\text {hot }}\left(R, R^{\prime}, v\right)\right] /|v| .
\end{aligned}
$$

In this equation, we assume the charge exchange rate is independent on the atom velocity $v$. Discretizing $R$ to be $R_{0, \text { in }}=R_{0}, R_{1}, R_{2}, \ldots, R_{i}, \ldots, R_{N-1}=R_{0, \text { out }}$, and replacing the integrals over $R$ in Eqs. (A1) and (A2) by summations, we write the charge exchange flux $\Phi_{\mathrm{CX} i}$, which is the representative value of the flux distributions at $R_{i}$, as the following form:

$$
\Phi_{\mathrm{CX} i}=\sum_{j}^{N-1} X_{i j} \Phi^{\mathrm{D}}{ }_{\mathrm{H} 2 j}+\sum_{j}^{N-1} Y_{i j} \Phi_{\mathrm{CX} j}
$$

where $\Phi^{\mathrm{D}}{ }_{\mathrm{H} 2 j}$ is the representative value of $\Phi^{\mathrm{D}}{ }_{\mathrm{H} 2}(R)$ at $R=R_{\mathrm{j}}$. $X_{i j}$ and $Y_{i j}$ are the coefficients for the summations, which are written as follows from Eqs. (7), (A1), and (A2):

$$
\begin{gathered}
X_{i j}=\left\{\begin{array}{ll}
C_{i} \int_{-\infty}^{0} f_{\mathrm{H}}\left(v, T_{\mathrm{dis}}\right) \exp \left[-\sum_{k=j}^{i}\left(S_{k}+C_{k}\right) \Delta R_{k} / v\right] \mathrm{d} v \Delta R_{j} / \Delta R_{i} & \left(R_{j}<R_{i}\right) \\
C_{i} \int_{0}^{\infty} f_{\mathrm{H}}\left(v, T_{\mathrm{dis}}\right) \exp \left[-\sum_{k=i}^{j}\left(S_{k}+C_{k}\right) \Delta R_{k} / v\right] \mathrm{d} v \Delta R_{j} / \Delta R_{i} & \left(R_{j}>R_{i}\right)
\end{array},\right. \\
Y_{i j}= \begin{cases}C_{\mathrm{i}} \int_{-\infty}^{0} f_{\mathrm{M}}\left(v, T_{\mathrm{p} j}\right) \exp \left[-\sum_{k=j}^{i}\left(S_{k}+C_{k}\right) \Delta R_{k} / v\right] \mathrm{d} v \Delta R_{j} / \Delta R_{i} & \left(R_{j}<R_{i}\right) \\
C_{\mathrm{i}} \int_{0}^{\infty} f_{\mathrm{M}}\left(v, T_{\mathrm{p} j}\right) \exp \left[-\sum_{k=i}^{j}\left(S_{k}+C_{k}\right) \Delta R_{k} / v\right] \mathrm{d} v \Delta R_{j} / \Delta R_{i} & \left(R_{j}>R_{i}\right)\end{cases}
\end{gathered}
$$

where $C_{i}, S_{i}$, and $T_{\mathrm{p} i}$ are the representative values of the $C_{\mathrm{H}}(R), S_{\mathrm{H}}(R)$, and $T_{\mathrm{p}}(R)$, respectively, and $\Delta R_{i}$ is the length of the space represented by $R_{i}$. The integrations over $v$ in the above equations are carried out numerically. Since Eq. (A3) is a system of linear equations for $\Phi_{\mathrm{CX}}$ i, the solutions are numerically calculated with known values of $\Phi^{\mathrm{D}}{ }_{\mathrm{H} 2}(R), X_{i j}$, and $Y_{i j}$. Once the $\Phi_{\mathrm{CX}}$ is obtained, the spatial and velocity distribution of the hot atoms $n_{\mathrm{H} \text { hot }}(R, v)$ is calculated with the same form to Eqs. (A1) and (8). 
${ }^{1}$ D. H. McNeill, J. Nucl. Mater. 162-164, 476 (1989).

${ }^{2}$ U. Samm and TEXTOR-94 Team, Plasma Phys. Controlled Fusion 41, B57 (1999).

${ }^{3}$ F. Wagner and U. Stroth, Plasma Phys. Controlled Fusion 35, 1321 (1993).

${ }^{4}$ N. Asakura, K. Shimizu, N. Hosogane, K. Itami, S. Tsuji, and M. Shimada, Nucl. Fusion 35, 381 (1995).

${ }^{5}$ J. L. Weaver, B. L. Welch, H. R. Griem, J. Terry, B. Lipschultz, C. S. Pitcher, S. Wolfe, D. A. Pappas, and C. Boswell, Rev. Sci. Instrum. 71, 1664 (2000)

${ }^{6}$ T. Shikama, S. Kado, H. Zushi, M. Sakamoto, A. Iwamae, and S. Tanaka, Phys. Plasmas 11, 4701 (2004).

${ }^{7}$ A. Iwamae, M. Hayakawa, M. Atake, M. Goto, S. Morita, and T. Fujimoto, Phys. Plasmas 12, 042501 (2005).

${ }^{8}$ A. Iwamae, A. Sakaue, N. Neshi, J. Yanagibayashi, M. Hasuo, M. Goto, and S. Morita, J. Phys. B 43, 144019 (2010).

${ }^{9}$ T. Fujimoto, K. Sawada, K. Takahata, K. Eriguchi, H. Suemitsu, K. Ishii, R. Okasaka, H. Tanaka, T. Maekawa, Y. Terumichi, and S. Tanaka, Nucl. Fusion 29, 1519 (1989).

${ }^{10}$ K. Sawada, K. Eriguchi, and T. Fujimoto, J. Appl. Phys. 73, 8122 (1993).

${ }^{11}$ K. Sawada and T. Fujimoto, J. Appl. Phys. 78, 2913 (1995).

${ }^{12}$ A. Pospieszczyk, Ph. Mertens, A. Huber, D. Reiter, D. Rusbüldt, B. Schweer, E. Vietzke, P. T. Greenland, and G. Sergienko, J. Nucl. Mater. 266, 138 (1999).

${ }^{13}$ U. Fantz, B. Heger, D. Wünderlich, and P. Pugno, J. Nucl. Mater. 313, 743 (2003).

${ }^{14}$ B. P. Lavrov, A. A. Solovév, and M. V. Tyutchev, J. Appl. Spectrosc. 32, 316 (1980).

${ }^{15}$ T. Shikama, S. Kado, H. Zushi, and S. Tanaka, Phys. Plasmas 14, 072509 (2007).

${ }^{16}$ K. Fujii, K. Mizushiri, T. Nishioka, T. Shikama, A. Iwamae, M. Goto, S. Morita, S. Kado, K. Sawada, and M. Hasuo, Rev. Sci. Instrum. 81, 033106 (2010).
${ }^{17}$ K. Fujii, T. Shikama, A. Iwamae, M. Goto, S. Morita, and M. Hasuo, Plasma Fusion Res. 5, S2079 (2010).

${ }^{18}$ K. Narihara, I. Yamada, H. Hayashi, and K. Yamauchi, Rev. Sci. Instrum. 72, $1122(2001)$.

${ }^{19}$ J. D. Hey, M. Korten, Y. T. Lie, A. Pospieszczyk, D. Rusbüldt, B. Schweer, B. Unterberg, J. Wienbeck, and E. Hintz, Contrib. Plasma Phys. 36, 583 (1996).

${ }^{20}$ U. Samm, P. Bogen, H. Hartwig et al., J. Nucl. Mater. 162, 24 (1989).

${ }^{21}$ T. Shikama, K. Fujii, K. Mizushiri, M. Hasuo, S. Kado, and H. Zushi, Plasma Phys. Controlled Fusion 51, 122001 (2009).

${ }^{22}$ S. Brezinsek, G. Sergienko, A. Pospieszczyk et al., Plasma Phys. Controlled Fusion 47, 615 (2005).

${ }^{23}$ I. Tobias and J. Vanderslice, J. Chem. Phys. 35, 1852 (1961).

${ }^{24}$ G. H. Dieke and R. W. Blue, Phys. Rev. 47, 261 (1935).

${ }^{25}$ A. V. Phelps, J. Phys. Chem. Ref. Data 19, 653 (1990).

${ }^{26}$ K. Fujii, K. Mizushiri, T. Nishioka, T. Shikama, A. Iwamae, M. Goto, S. Morita, and M. Hasuo, Nucl. Instrum. Methods Phys. Res., Sec. A 623, 690 (2010).

${ }^{27}$ S. Masuzaki, T. Morisaki, N. Ohyabu, A. Komori, H. Suzuki, N. Noda, Y. Kubota, R. Sakamoto, K. Narihara, K. Kawahata, K. Tanaka, T. Tokuzawa, S. Morita, M. Goto, M. Osakabe, T. Watanabe, Y. Matsumoto, O. Motojima, and LHD Experimental Group, Nucl. Fusion 42, 750 (2002).

${ }^{28}$ P. S. Krstic and D. R. Schultz, J. Phys. B 36, 385 (2003).

${ }^{29}$ M. Misakian and J. C. Zorn, Phys. Rev. A 6, 2180 (1972).

${ }^{30}$ S. Tamor, J. Comput. Phys. 40, 104 (1981).

${ }^{31}$ R. K. Janev, W. D. Langer, Jr., K. Evans et al., Elementary Processes in Hydrogen-Helium Plasmas (Springer 1987).

${ }^{32}$ D. Reiter, P. Bogen, and U. Samm, J. Nucl. Mater. 196, 1059 (1992).

${ }^{33}$ T. Takagi, Phys. Scr. T96, 52 (2002). 